\title{
Finite-width effects in unstable-particle production at hadron colliders
}

\author{
P. Falgari, ${ }^{a}$ A.S. Papanastasiou ${ }^{b}$ and A. Signer ${ }^{c, d}$ \\ ${ }^{a}$ Institute for Theoretical Physics and Spinoza Institute, Utrecht University, \\ Leuvenlaan 4, 3508 TD Utrecht, The Netherlands \\ ${ }^{b}$ DESY, Deutsches Elektronen-Synchrotron, \\ Notkestraße 85, D-22607 Hamburg, Germany \\ ${ }^{c}$ Laboratory for Particle Physics (LTP), Paul Scherrer Institut, \\ CH-5232 Villigen PSI, Switzerland \\ ${ }^{d}$ Institute for Theoretical Physics, University of Zurich, \\ Winterthurerstrasse 190, CH-8057 Zurich, Switzerland \\ E-mail: p.falgari@uu.nl, andrew.papanastasiou@desy.de, \\ adrian.signer@psi.ch
}

ABSTRACT: We present a general formalism for the calculation of finite-width contributions to the differential production cross sections of unstable particles at hadron colliders. In this formalism, which employs an effective-theory description of unstable-particle production and decay, the matrix element computation is organized as a gauge-invariant expansion in powers of $\Gamma_{X} / m_{X}$, with $\Gamma_{X}$ and $m_{X}$ the width and mass of the unstable particle. This framework allows for a systematic inclusion of off-shell and non-factorizable effects whilst at the same time keeping the computational effort minimal compared to a full calculation in the complex-mass scheme. As a proof-of-concept example, we give results for an NLO calculation of top-antitop production in the $q \bar{q}$ partonic channel. As already found in a similar calculation of single-top production, the finite-width effects are small for the total cross section, as expected from the naïve counting $\sim \Gamma_{t} / m_{t} \sim 1 \%$. However, they can be sizeable, in excess of $10 \%$, close to edges of certain kinematical distributions. The dependence of the results on the mass renormalization scheme, and its implication for a precise extraction of the top-quark mass, is also discussed.

Keywords: NLO Computations, Hadronic Colliders

ARXIV EPRINT: 1303.5299 


\section{Contents}

1 Introduction 1

2 Effective-theory description of unstable-particle production 4

2.1 Born amplitudes 5

$\begin{array}{lll}2.2 & \text { Virtual corrections } & 7\end{array}$

2.3 Real corrections 9

$\begin{array}{lll}2.4 \text { Cross sections } & 11\end{array}$

2.5 Mass scheme 11

3 Top-antitop production at hadron colliders $\quad 12$

$\begin{array}{ll}3.1 & \text { Leading tree-level amplitude } \\ & 13\end{array}$

$\begin{array}{lll}3.2 & \text { Sub-leading tree-level contributions } & 14\end{array}$

$\begin{array}{ll}3.3 \text { Virtual QCD corrections } & 16\end{array}$

$\begin{array}{lll}3.4 & \text { Real QCD corrections } & 20\end{array}$

3.5 Mass-scheme choice 21

3.6 Validity of EFT results 23

4 Results $\quad 24$

4.1 Invariant and transverse masses 25

$\begin{array}{ll}4.2 & \text { Individual NLO contributions } 28\end{array}$

$\begin{array}{lll}4.3 & \text { Forward-backward asymmetry } & 29\end{array}$

$\begin{array}{lll}4.4 & \text { Pole mass versus PS mass } & 29\end{array}$

5 Conclusions $\quad 34$

\section{Introduction}

The performance of the Large Hadron Collider (LHC) so far has been extremely successful, with about $5 \mathrm{fb}^{-1}$ of integrated luminosity collected in the 2011 run and more than $20 \mathrm{fb}^{-1}$ in 2012 . This has led to an unprecedented accuracy in the measurements of many Standard Model (SM) cross sections and distributions, both in the electroweak and strong sectors, and also to the discovery of a new particle consistent with a SM Higgs boson. With the high-energy run which will follow the 2013 shutdown, experimental errors are bound to reduce even further, leading to higher-precision measurements and to the possible observation of new-physics effects beyond the SM. This high experimental precision clearly motivates similarly accurate theoretical predictions for cross sections and kinematical distributions, so that on the one hand the clean extraction of signals from the data is possible and, on the other hand, contributions to the backgrounds to processes of interest can be accurately constrained. 
Most of the phenomenologically interesting processes at the LHC, such as $W$ and $Z$ boson production, top-quark production and Higgs production, not to mention beyondthe-Standard-Model (BSM) processes, like supersymmetric (SUSY) particle production, involve massive unstable particles. These particles are not asymptotic states and show up in detectors as energetic jets and leptons, often being accompanied by large transverse missing energy $\mathbb{E}_{T}$. The necessity of precise theoretical predictions therefore raises the question of how to correctly treat the decay of the intermediate unstable particle to the physical final states. For observables which are inclusive in the final states originating from the unstable-particle decay, it is often sufficient to treat the massive particles as stable, ignoring their decay. The error associated with this approximation is formally of order $\Gamma_{X} / m_{X}$, where $\Gamma_{X}$ and $m_{X}$ are the width and mass of the unstable particle. For the aforementioned processes this corresponds to less than a few percent, i.e. typically smaller than the experimental errors. Clearly, while the stable approximation is appropriate for the total cross section, it cannot be used to predict arbitrary kinematical observables. Moreover, the error associated with this approximation could actually be numerically sizeable for new, yet undiscovered wide resonances, for example strongly-decaying SUSY particles.

A step forward towards a realistic description of production and decay of an unstable particle $X$ is the narrow-width approximation (NWA) which is a framework commonly used in the context of high-energy calculations for hadron colliders. In the NWA the particle is produced and allowed to decay to the physical final states while remaining on shell. At next-to-leading order (NLO), radiative corrections are given by factorizable virtual and real contributions to the on-shell production and decay subprocesses. While technically only slightly more involved than the stable-top approximation, the NWA preserves spin correlations between the production and decay subprocesses, and allows for realistic kinematical cuts on the momenta of the physical final states (i.e. leptons and jets).

The NWA includes neither off-shell effects related to the virtuality of the intermediate unstable-particle propagator, nor non-factorizable corrections linking the production and decay subprocesses. Sub-resonant or non-resonant contributions, which correspond to diagrams with the correct physical final state but which involve fewer or no intermediate unstable-particle propagators, are also neglected. As in the stable approximation, these finite-width effects are expected to be small, of order $\Gamma_{X} / m_{X}$, for inclusive-enough observables. This follows from the suppression of the non-resonant contributions and from large cancellations between virtual and real non-factorizable corrections, with the width acting as a natural infrared regulator whose dependence largely cancels in infrared safe quantities [1]. However, for arbitrary kinematical distributions and in particular, close to certain kinematical thresholds where the cancellations mentioned above are less effective, finite-width effects can be large.

Strikingly, in refs. [2-4] it was pointed out that the naïve expectation of the error associated with the NWA can be underestimated by an order of magnitude for BSM processes where the mass of daughter particles approaches the mass of the parent particle $X$. This is relevant for searches of SUSY in decay cascades, where one often observes some degree of mass degeneracy between particles in different steps of the cascade. Recently, non-negligible off-shell effects $(\sim 10 \%)$ were observed even in light Higgs production and decay to massive 
vector bosons [5, 6], and shown to arise from Higgs-continuum interference at large values of the boson-pair invariant mass. While these off-shell effects can be suppressed by means of suitable experimental cuts, and are therefore not relevant to present Higgs measurements at the LHC, they nonetheless show that the error associated with the NWA can be significantly larger than its naïve estimate. Thus, it is clear that an approach that goes beyond the NWA and which includes at least the dominant finite-width effects is desirable. Note that for a heavy Higgs the interference of resonant and non-resonant terms is large due to the large value of the ratio $\Gamma_{H} / m_{H}$. In this case the NWA is expected to be a poor approximation.

A possible solution to the issue of finite-width effects is clearly the calculation of the full, gauge-invariant set of diagrams corresponding to a given physical final state. This approach includes the coherent sum of resonant and non-resonant contributions, treats the intermediate resonant particles as fully off-shell and contains both factorizable and non-factorizable corrections at NLO. Self-energy contributions can be resummed in the unstable-particle propagator in a consistent gauge-invariant way using, for example, the complex-mass scheme $[7,8]$. Examples applying the complex-mass scheme to production of unstable particles at NLO include the calculations of four-fermion production at an $e^{+} e^{-}$ collider [7], Higgs decay to vector-boson pairs [9] and two recent independent calculations of off-shell effects in $t \bar{t}$ production [10-12]. While the complex-mass scheme approach is completely general and very flexible, allowing the calculation of arbitrary kinematical distributions, the full NLO computation is technically challenging, requiring both the calculation of a much larger set of diagrams than for the corresponding on-shell process and special techniques to handle 5- or 6-point functions with complex masses.

An alternative approach to the full NLO calculation was presented in ref. [13] and applied to processes of $t$-channel and $s$-channel single-top production $[13,14]$. The approach of ref. [13] is the generalization of the effective field theory (EFT) description of resonant-particle production of ref. [15], which was employed in the calculation of inclusive $W$-pair production at an $e^{+} e^{-}$collider $[16,17]$. The EFT calculation results in a systematic, gauge-invariant expansion of the matrix elements in powers of $\Gamma_{X} / m_{X}$, in a way which can be considered a generalization of the pole approximation [18, 19]. Compared to the full NLO calculation in the complex-mass scheme, the effective-theory approach has the advantage of identifying the terms that are relevant to achieving a given target accuracy prior to the actual calculation. This greatly reduces the complexity of the computation while at the same time allows for the inclusion of the leading off-shell and non-factorizable effects in a completely differential manner. For single-top production finite-width effects were found to be small for inclusive-enough observables, although they can be large close to the kinematical edges of some distributions. This general picture is consistent with the results found by the full NLO calculations of top-pair production [20].

In this paper we give a second example of the application of the EFT formalism of ref. [13] and calculate the cross section for the pair-production process $q \bar{q} \rightarrow t \bar{t} \rightarrow$ $W^{+} W^{-} b \bar{b}$. The top-pair production process has been studied extensively over the years in the stable-top and narrow-width approximations and, more recently, using the complexmass scheme (a detailed list of references is given in section 3). It thus represents a perfect proof-of-concept calculation by which to test the validity of the EFT formalism, extend 
it to more than one unstable particle and to compare it to different available approaches. The paper is organized as follows: in section 2 we review the effective-theory formalism and introduce a treatment of real corrections which differs slightly from the one used in ref. [13]. The calculation of the LO and NLO relevant amplitudes for the specific example of $t \bar{t}$ production is described in section 3. In section 4 we present results for several distributions and assess the effect of finite-width contributions by comparing the effectivetheory predictions with results obtained in the NWA. In that same section we also discuss the effects of using different mass-renormalization schemes (more precisely the pole and PS schemes) on the cross section and distributions, and their possible implications for a precise extraction of the top-quark mass from data. Finally, our conclusions are given in section 5 .

\section{Effective-theory description of unstable-particle production}

The effective-theory framework for the description of unstable-particle production used in this work was first formulated for the total cross section in ref. [15] and applied to the case of inclusive $W^{+} W^{-}$production at an $e^{+} e^{-}$collider in refs. [16, 17]. The formalism was later extended to the more general case of differential cross sections and applied to singletop production at hadron colliders $[13,14]$. In this section we review the main features of this approach, referring the reader to the aforementioned references for further details.

Unstable-particle effective theory is built upon the hierarchy of two scales, namely, the typical virtuality of the resonant unstable particle $X$, which is set by its decay width, $p_{X}^{2}-m_{X}^{2} \sim m_{X} \Gamma_{X}$, and the particle mass $m_{X}$. This hierarchy is encoded in the ratio $\Gamma_{X} / m_{X} \ll 1$. The latter is treated as a small parameter, $\delta$, in line with the strong and electroweak coupling constants $\alpha_{s}$, and $\alpha_{e w}$ and allows for a systematic expansion of the full matrix elements. These different expansion parameters are related by the counting scheme ${ }^{1}$

$$
\frac{\Gamma_{X}}{m_{X}} \sim \alpha_{s}^{2} \sim \alpha_{e w}
$$

In the following we will generically refer to any of the above parameters as $\delta$. The expansion in $\delta$ is implemented at the Lagrangian level, replacing the (B)SM fields and interactions with effective fields and vertices. The effective fields are associated with different momentum regions defined according to the scaling of their momenta with respect to the parameter $\delta$ and encode the physics at the two very different scales that characterize the production and decay process. ${ }^{2}$ For the problem at hand these momentum regions are a hard region $\left(q_{0} \sim|\vec{q}| \sim m_{X}\right)$, a soft region $\left(q_{0} \sim|\vec{q}| \sim m_{X} \delta\right)$ and collinear regions $\left(n_{i} \cdot q \sim m_{X} \delta, \bar{n}_{i} \cdot q \sim m_{X}, q_{\perp} \sim m_{X} \sqrt{\delta}\right)$. Here $n_{i}, \bar{n}_{i}$ are light-like vectors associated with the momenta of the external massless particles, $n_{i}=\left(1, \vec{p}_{i} /\left|\vec{p}_{i}\right|\right), \bar{n}_{i}=\left(1,-\vec{p}_{i} /\left|\vec{p}_{i}\right|\right)$, and $q_{\perp}$ is the remaining, perpendicular component of the momentum $q$.

In the effective theory only low-virtuality modes with $p^{2} \lesssim m_{X}^{2} \delta$ are kept as dynamical degrees of freedom, and are described by effective fields in the Lagrangian. These include,

\footnotetext{
${ }^{1}$ Note that in (2.1) we assume that the unstable-particle decay proceeds via electroweak decay channels, $\Gamma_{X} \propto \alpha_{e w}$. For resonances decaying via strong interactions the counting scheme is $\Gamma_{X} \sim \alpha_{s} \sim \sqrt{\alpha_{e w}}$.

${ }^{2}$ Here and in the following we assume that the invariants $s_{i j}=2 p_{i} \cdot p_{j}$ constructed from the external momenta are of the same order of $m_{X}^{2}$, and we treat them as a single scale.
} 
in particular, a field $\Phi_{X}$ to describe the resonant unstable particle $X$. The finite-width of the particle is resummed into the leading EFT kinetic term in a generalisation of the heavy-quark effective theory (HQET) Lagrangian in the case of a non-vanishing width [15],

$$
\mathcal{L}_{\mathrm{EFT}, k i n}^{(0)}=2 \hat{m}_{X} \Phi_{x}^{\dagger}\left(i v \cdot \partial-\frac{\Omega_{X}}{2}\right) \Phi_{X}
$$

where $\hat{m}_{X} v$, with $v^{2}=1$, represents the large, on-shell component of the resonant-particle momentum, and $\hat{m}_{X}$ is the renormalized mass in a generic renormalization scheme. The coefficient $\Omega_{X}$ is related to the complex pole $\mu_{X}^{2} \equiv m_{X}^{2}-i m_{X} \Gamma_{X}$ of the full unstable-particle propagator,

$$
\Omega_{X}=\frac{\mu_{X}^{2}-\hat{m}_{X}^{2}}{\hat{m}_{X}}
$$

In the pole scheme, $\hat{m}_{X}=m_{X}, \Omega_{X}$ has the simple form $\Omega_{X}=-i \Gamma_{X}$. Additional terms in the EFT Lagrangian are given by bilinear terms for soft and collinear fields, powersuppressed corrections to (2.2) and terms describing the interaction of $\Phi_{X}$ and collinear fields with soft fields. Hard modes are not explicitly part of the effective Lagrangian and their contribution is encoded in matching coefficients multiplying effective interaction vertices. These can be schematically parameterized as

$$
\mathcal{C}_{i, P}\left(\mu_{X}\right) \mathcal{F}_{P}^{i}\left(\Phi_{X}^{\dagger}, \phi_{c}, \phi_{s}, \partial_{\mu}\right), \quad \mathcal{C}_{j, D}\left(\mu_{X}\right) \mathcal{F}_{D}^{j}\left(\Phi_{X}, \phi_{c}, \phi_{s}, \partial_{\mu}\right), \quad \mathcal{C}_{k, N R}\left(\mu_{X}\right) \mathcal{F}_{N R}^{k}\left(\phi_{c}, \phi_{s}, \partial_{\mu}\right),
$$

where $\mathcal{F}_{P}^{i}, \mathcal{F}_{D}^{j}$ and $\mathcal{F}_{N R}^{k}$ denote functions of fields and derivatives and the indices $i, j, k$ label different Lorentz structures. $\phi_{c, s}$ generically represent collinear and soft fields and $\mathcal{C}_{i, P}$ and $\mathcal{C}_{j, D}$ are the hard matching coefficients of the production and decay effective vertices, which are computed from on-shell SM amplitudes. In this context "on-shell" has to be understood as $p_{X}^{2}=\hat{m}_{X}^{2}+\hat{m}_{X} \Omega_{X}=\mu_{X}^{2}$, meaning that the effective couplings in the Lagrangian are in general complex. This is a feature that the EFT framework shares with the complex-mass scheme. The interaction terms $\mathcal{C}_{k, N R} \mathcal{F}_{N R}^{k}$ encode the contribution of non-resonant configurations which also contribute to the cross section starting from a certain order in $\delta$. Note that in order to describe pair-production of unstable particles, e.g. $t \bar{t}$ production, two distinct resonant fields, $\Phi_{t}$ and $\Phi_{\bar{t}}$, have to be introduced which annihilate a top and an antitop state respectively. Furthermore, the kinetic terms will contain two velocities $v$ and $\bar{v}$ which are generally different.

\subsection{Born amplitudes}

From a practical point of view, at tree-level the EFT result coincides with an expansion of the matrix element around the complex pole of the propagator. In fact, the EFT approach can be viewed as a generalization, to arbitrary order in $\delta$, of the pole approximation $[18,19]$. To be specific, let us consider the process

$$
i_{1}\left(p_{1}\right) i_{2}\left(p_{2}\right) \rightarrow X\left(p_{X}\right), \ldots \rightarrow f_{1}\left(k_{1}\right) f_{2}\left(k_{2}\right)
$$

where the final-state particles $f_{1}$ and $f_{2}$ can originate from the decay of an intermediate unstable scalar particle $X$ (the generalization to higher spins is trivial), or from other production mechanisms. The Feynman-diagram topologies contributing to this process at the 


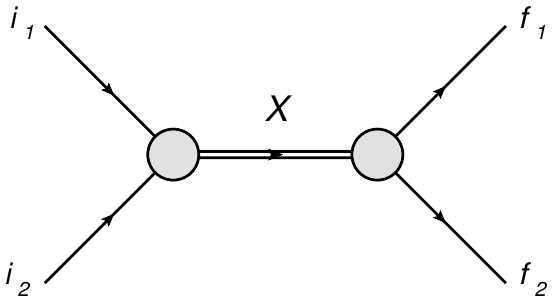

(a)

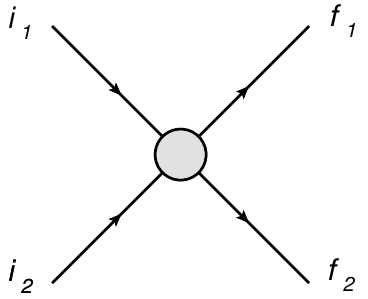

(b)

Figure 1. Feynman-diagram topologies contributing to the process $i_{1} i_{2} \rightarrow f_{1} f_{2}$. (a) resonant production through an intermediate unstable $X$; (b) non-resonant production.

tree-level are given by the resonant and non-resonant contributions drawn in figure 1, where the grey blobs denote the model-dependent production and decay vertices. The tree-level amplitude can be written as

$$
\mathcal{A}_{\text {tree }}=\frac{\mathcal{V}_{P}\left(\left\{p_{i}\right\}, p_{X}\right) \mathcal{V}_{D}\left(p_{X},\left\{k_{i}\right\}\right)}{p_{X}^{2}-m_{0, X}^{2}}+\mathcal{N}\left(\left\{p_{i}\right\},\left\{k_{i}\right\}\right),
$$

where $\mathcal{V}_{P}$ and $\mathcal{V}_{D}$ represent the vertices for the production and decay of the (off-shell) particle $X$ respectively, while $\mathcal{N}$ contains the non-resonant contributions. $m_{0, X}$ denotes the bare mass of the particle $X$. It is immediately clear from eq. (2.6) that due to the intermediate resonant propagator, $p_{X}^{2}-m_{0, X}^{2} \sim m_{X}^{2} \delta$, only resonant diagrams will contribute to the amplitude at leading order in $\delta$, while $\mathcal{N}$ will generally be suppressed by extra powers of $\delta$. The actual suppression of the non-resonant contributions is determined from the interplay between the suppression from the missing resonant propagators and the scaling of the couplings appearing in $\mathcal{V}_{P}, \mathcal{V}_{D}$ and $\mathcal{N}$, see eq. (2.1).

The gauge-invariant expansion in $\delta$ of the amplitude, eq. (2.6), is obtained by expanding the matrix elements for production and decay around $p_{X}^{2}=\mu_{X}^{2}$. This requires a projection of the external momenta $\left\{p_{i}\right\},\left\{k_{i}\right\}$ onto on-shell configurations $\left\{\bar{p}_{i}\right\},\left\{\bar{k}_{i}\right\}$, with

$$
\bar{p}_{i}=\bar{p}_{i}\left(p_{i}, p_{X}\right) \quad \bar{k}_{i}=\bar{k}_{i}\left(k_{i}, p_{X}\right) .
$$

The on-shell projection is chosen such that momentum is exactly conserved at each vertex and $\bar{p}_{X}^{2}=\mu_{X}^{2}$. Note that the explicit form of the projection in eq. (2.7) is not unique, being defined up to terms of order $\delta$. However, calculations at order $\delta^{n}$ obtained with different projections, deviate from each other by sub-leading corrections of order $\delta^{n+1}$, i.e. always an order higher than the target accuracy of the calculation.

Adopting eq. (2.7), the expansion in $\delta$ of the tree-level matrix element reads

$$
\begin{aligned}
\mathcal{A}_{\text {tree }}= & \frac{\mathcal{A}_{P} \mathcal{A}_{D}}{\Delta_{X}} \\
& +\frac{1}{\Delta_{X}}\left(\left(p_{i}-\bar{p}_{i}\right) \frac{\partial \mathcal{V}_{P}}{\partial p_{i}} \mathcal{A}_{D}+\mathcal{A}_{P}\left(k_{i}-\bar{k}_{i}\right) \frac{\partial \mathcal{V}_{D}}{\partial k_{i}}\right)+\mathcal{N}+\ldots
\end{aligned}
$$

where $\mathcal{A}_{P} \equiv \mathcal{V}_{P}\left(\left\{\bar{p}_{i}\right\}, \bar{p}_{X}\right), \mathcal{A}_{D} \equiv \mathcal{V}_{D}\left(\bar{p}_{X},\left\{\bar{k}_{i}\right\}\right)$ are evaluated with the projected momenta $\bar{p}_{i}, \bar{k}_{i}$, and the ellipses represent higher-order terms in $\delta$. As expected, the leading-order 

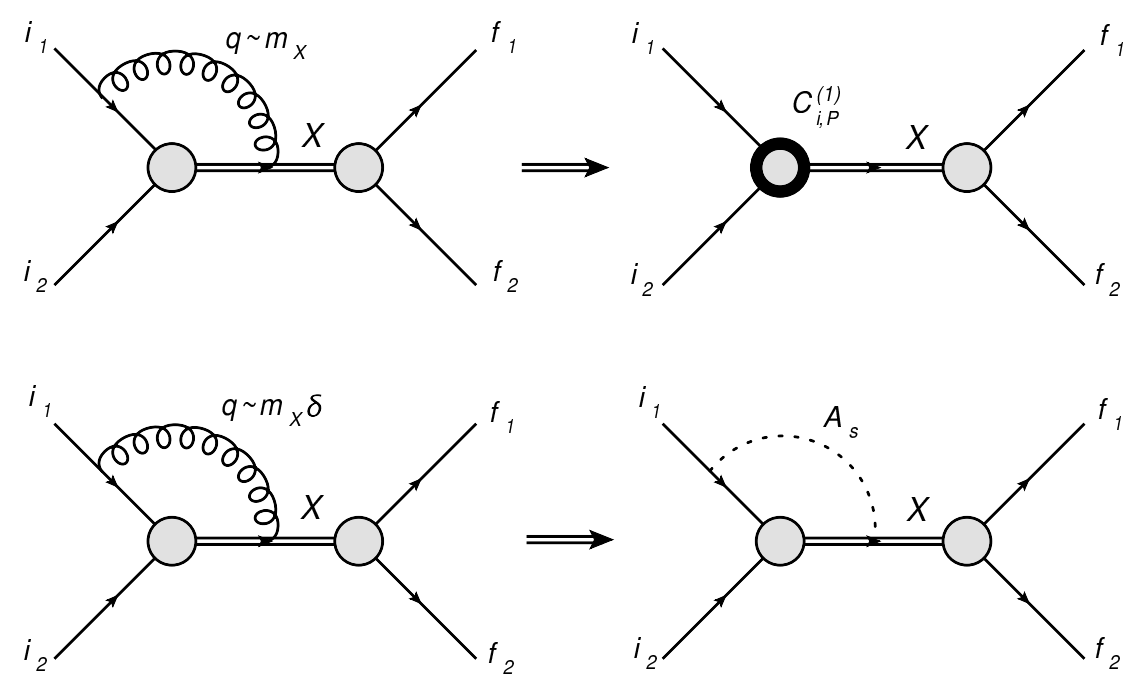

Figure 2. Correspondence between the expansion by regions and the EFT calculation: the hard-region contribution (top left) corresponds to a $\mathcal{O}\left(\alpha_{s}\right)$ correction to the production matching coefficient $C_{i, P}$ (top right), while the soft-region contribution (bottom left) reproduces one-loop soft-gluon corrections in the EFT (bottom right).

amplitude (first line in eq. (2.8)) corresponds to the resonant diagram with the vertices for production and decay of the unstable particle replaced by (gauge-invariant) on-shell amplitudes. These amplitudes are in fact directly related to the matching coefficients $C_{0, P}$, $C_{0, D}$ of the (leading) effective vertices appearing in eq. (2.4). The modified propagator $\Delta_{X} \equiv p_{X}^{2}-\mu_{X}^{2}=p_{X}^{2}-\hat{m}_{X}^{2}-\hat{m}_{X} \Omega_{X}$ Dyson-resums the finite-width effects related to the self-energy $\Pi_{X}\left(p_{X}^{2}\right)$ of the particle $X$ :

$$
\frac{1}{p_{X}^{2}-\hat{m}_{X}^{2}+\Pi_{X}\left(p_{X}^{2}\right)}=\frac{1}{\Delta_{X}}+\ldots
$$

with higher-order terms in $\delta$ indicated by the ellipses. Note that it is not the full self-energy that is kept in the propagator. Only the gauge-invariant hard part of the self-energy $\Omega_{X}$ contributes to the matching coefficient and is resummed in the propagator. The sub-leading gauge-violating residual soft terms are included perturbatively and are combined with other contributions to form a separate gauge-invariant part of the one-loop amplitude. The next term in $\delta$ in the expansion of the amplitude (second line in eq. (2.8)) receives contributions from non-resonant diagrams, as expected, but also from resonant ones in which the propagator $\Delta_{X}$ is cancelled by terms of the form $p_{i}-\bar{p}_{i} \sim \delta$ and $k_{i}-\bar{k}_{i} \sim \delta$, which originate from the expansion around on-shell configurations. In the EFT language these contributions are described by effective four-particle operators, i.e. terms of the form $\mathcal{C}_{k, N R} \mathcal{F}_{N R}^{k}$ in eq. (2.4). We stress that while the leading resonant term is gauge-invariant, at higher orders in $\delta$ only the sum of resonant and non-resonant contributions is on the whole gauge independent.

\subsection{Virtual corrections}

Virtual corrections in the EFT framework are divided into two categories: loop corrections to the EFT matrix elements, where the degrees of freedom flowing in the loops are 
given by the effective fields $\Phi_{X}, \phi_{c}, \phi_{s}$, and corrections to the hard matching coefficients $C_{i, P}, C_{j, D}, C_{k, N R}$. The latter are computed by a "matching procedure" which arises by requiring that the full-theory matrix element and the EFT result coincide order by order in $\delta$. They can be written as a power series in the couplings (the QCD coupling, in this case),

$$
C_{i, P}=C_{i, P}^{(0)}+\frac{\alpha_{s}}{2 \pi} C_{i, P}^{(1)}+\ldots
$$

with similar expressions for $C_{j, D}$ and $C_{k, N R}$. In practice, the EFT matrix elements and the matching coefficients can be obtained from an expansion of SM matrix elements using the method of regions [21], which we find to be more convenient here than an explicit two-step calculation of matching coefficients and effective-theory matrix elements. This is the approach we adopt for the calculation of the amplitudes for $t \bar{t}$ production in section 3 , where details on how the expansion by regions is implemented for the specific case of resonant top-pair production are given.

As already outlined, the momentum regions relevant to the expansion are the hard, soft and collinear regions. The contributions of hard momenta $\left(q \sim m_{X}\right)$ correspond to the matching coefficients, while those from an expansion in the soft region $\left(q \sim m_{X} \delta\right)$ of the full SM integrals reproduces loop contributions in the effective theory. This is schematically depicted in figure 2 for the case of the one-loop gluonic correction to the production vertex. Hard corrections correspond to factorizable contributions to the production or decay subprocesses [22], and, at leading order in $\delta$, coincide with the matrix element for production or decay of the on-shell massive particle(s). On the other hand, soft corrections encode non-factorizable interferences between the production and decay subprocesses as well as off-shell effects. Note that in general the contribution from collinear regions is needed to reproduce the full SM matrix element. However, for the case considered, this contribution vanishes if loop integrals are regularized dimensionally and the external light-fermion masses are set to zero, as done in this work. It can thus be safely ignored in the following discussion. In the soft region, the unstable-particle propagator in the loop, $\left(p_{X}-q\right)^{2}-m_{X}^{2} \sim m_{X}^{2} \delta$, is resonant and has to be Dyson-resummed,

$$
\frac{1}{\left(p_{X}-q\right)^{2}-\hat{m}_{X}^{2}+\Pi_{X}\left(\left(p_{X}-q\right)^{2}\right)}=\frac{1}{\Delta_{X}-2 \bar{p}_{X} \cdot q}+\ldots,
$$

with the ellipses denoting, as usual, higher-order terms in $\delta$. As in the tree-level matrix element, only the on-shell gauge-invariant part of the self energy is resummed in the resonant propagator. In the hard region, where $\left(p_{X}-q\right)^{2}-\hat{m}_{X}^{2} \sim \hat{m}_{X}^{2} \gg m_{X} \Gamma_{X}$, no self-energy resummation is necessary and gauge-invariance is similarly preserved.

An important feature of the effective-theory approach is that the EFT counting scheme, eq. (2.1), enables one to assign the correct parametric scaling to any particular contribution of a Feynman diagram prior to its actual calculation. This determines whether or not it has to be computed to obtain a given accuracy in $\delta$.

Suppose for example that one wants to compute all terms which scale as $\alpha_{s} \sim \delta^{1 / 2}$ relative to the leading tree-level contribution. From now on we will refer to such corrections as NLO corrections. If we ignore the couplings appearing in the production and decay vertices $\mathcal{V}_{P}$ and $\mathcal{V}_{D}$, the leading tree-level matrix element (first term in eq. (2.8)) scales as 


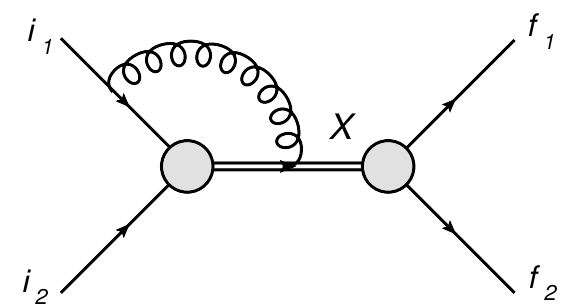

(a)

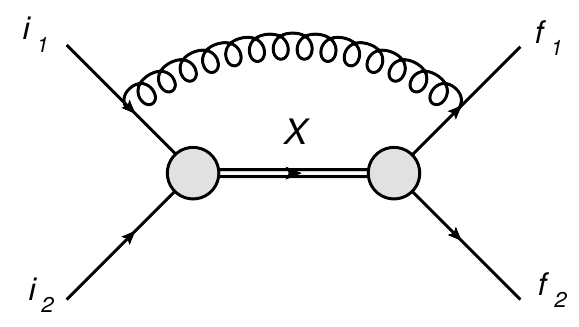

(b)

Figure 3. Examples of triangle and box diagrams contributing to the production and decay of the scalar $X$ at one loop.

$1 / \Delta_{X} \sim \delta^{-1}$, and NLO is thus defined as the sum of all terms scaling as $\delta^{-1} \delta^{1 / 2}=\delta^{-1 / 2}$. One can then immediately see that the sub-leading tree-level terms in eq. (2.8), which scale as $\delta^{-1} \delta=1$ (assuming that $\mathcal{V}_{P} \mathcal{V}_{D} \sim \mathcal{N}$ ), are not part of the NLO matrix element, contributing to the amplitude only at NNLO. Consider now the triangle and box integrals shown in figure 3. In the soft region of the loop momentum $\left(q \sim m_{X} \delta\right)$, the unstableparticle propagator inside the loop scales as $\delta^{-1}$, while the gluon and fermion propagators scale as $\delta^{-2}$ and $\delta^{-1}$ respectively. Taking into account the scaling of the volume element $d^{4} q \sim \delta^{4}$, one finds the scaling $\delta^{-1} \alpha_{s} \delta^{4} \delta^{-2} \delta^{-1} \delta^{-1} \sim \delta^{-1 / 2}$ for the soft part of the triangle, while the soft part of the box scales as $\alpha_{s} \delta^{4} \delta^{-2} \delta^{-1} \delta^{-1} \delta^{-1} \sim \delta^{-1 / 2}$. Therefore, the soft limit of both the triangle and box contribute at NLO. In the hard region $\left(q \sim m_{X}\right)$ all the propagators inside the loop scale parametrically as $\sim 1$ (in units of the mass $m_{X}$ ). Thus, the hard part of the triangle scales as $\delta^{-1} \alpha_{s} 1^{4} / 1^{3} \sim \delta^{-1 / 2}$, while for the box one finds $\alpha_{s} 1^{4} / 1^{4} \sim \delta^{1 / 2}$. In this case only the hard part of the triangle counts as NLO, while the hard box integral is highly suppressed, scaling as a $\mathrm{N}^{3} \mathrm{LO}$ correction. Given that for the simple process $(2.5)$, the hard box in figure $3(\mathrm{~b})$ is the most complicated integral at one loop, discarding it simplifies the NLO calculation. As we will see in section 3.3, for the phenomenologically relevant case of top-pair production one can use the same scaling arguments to show that all one-loop hard 5- and 6-point functions are parametrically suppressed and need not be computed to achieve NLO accuracy in the resonant region.

\subsection{Real corrections}

Even though the expansion by regions is well understood for loop diagrams, it is less clear how the expansion can in general be implemented for real corrections, since in the presence of an extra massless particle with momentum $q$, the expansion parameter can be $p_{X}^{2}-\mu_{X}^{2}$, $\left(p_{X}+q\right)^{2}-\mu_{X}^{2}$ or both. While the split into hard and soft contributions is possible for the total cross section, by relating real corrections to cut one-loop diagrams via the optical theorem, the formulation of the expansion in hard and soft contributions is not straightforward for an arbitrary observable. In refs. $[13,14]$ we proposed a way to circumvent this problem by using the exact real-radiation matrix element and expanding the integrated infrared and collinear subtraction terms in $\delta$ so as to properly match the singularity structure of the expanded virtual matrix element. 


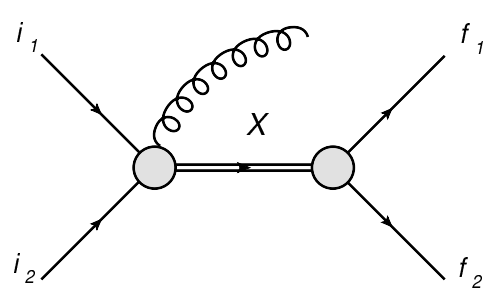

(a)

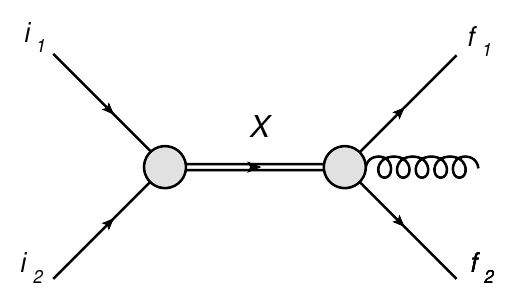

(b)

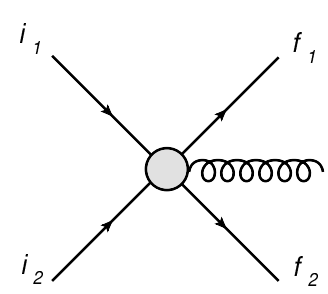

(c)

Figure 4. Real gluonic corrections to the process $i_{1} i_{2} \rightarrow f_{1} f_{2}$.

While this "ad-hoc" treatment of the real matrix element was sufficient to illustrate the basic features of the EFT approach and to assess the contribution of non-factorizable corrections in single-top production, a theoretically more satisfactory treatment of real corrections is certainly desirable. In particular, one would wish for a framework in which a strict expansion in $\delta$ for both virtual and real corrections is achieved and gauge-invariance is exact order-by-order in $\delta$. Here we introduce such a framework which will be used in section 3.4 for the calculation of real corrections to $t \bar{t}$ production.

Real gluon-emission contributions fall into three categories, as indicated in figure 4 . Topology (a) and (b) represent emission from the production and decay subprocess respectively, whereas topology (c) is due to generic non-resonant real-emission. Analogously to eq. (2.8), the corresponding amplitude can be written as

$$
\mathcal{A}_{\text {real }}=\frac{\mathcal{A}_{P+g} \mathcal{A}_{D}}{\Delta_{X}}+\frac{\mathcal{A}_{P} \mathcal{A}_{D+g}}{\Delta_{X g}}+\mathcal{A}_{\text {sub-lead }},
$$

where $\Delta_{X g}=\left(p_{X}+q\right)^{2}-\mu_{X}^{2}$ and $\mathcal{A}_{P+g}\left(\mathcal{A}_{D+g}\right)$ is the amplitude for on-shell production (decay) of the unstable particle with an additional real gluon. The first two terms in eq. (2.12) contribute to the leading resonant real-emission amplitude, whereas $\mathcal{A}_{\text {sub-lead }}$ contains terms sub-leading in $\delta$. Note that in this picture there is no topology with gluon emission from the unstable particle. In the corresponding Feynman diagram either of the two unstableparticle propagators can be resonant. In fact, when the emitted gluon is soft, $q \sim m_{X} \delta$, both propagators can simultaneously be resonant, $p_{X}^{2}-m_{0, X}^{2} \sim\left(p_{X}+q\right)^{2}-m_{0, X}^{2} \sim m_{X}^{2} \delta$. However, it can be shown [23] that the product of two unstable-particle poles can be written as the sum of two terms containing a single massive pole and a soft singularity

$$
\frac{1}{\left(p_{X}^{2}-m_{0, X}^{2}\right)\left(\left(p_{X}+q\right)^{2}-m_{0, X}^{2}\right)}=\frac{1}{2 p_{X} \cdot q}\left[\frac{1}{p_{X}^{2}-m_{0, X}^{2}}-\frac{1}{\left(p_{X}+q\right)^{2}-m_{0, X}^{2}}\right],
$$

so that the parameterization eq. (2.12) still holds true.

Following eq. (2.12), the squared amplitude, $\mathcal{M}_{\text {real }} \equiv\left|\mathcal{A}_{\text {real }}\right|^{2}$, can be written as

$$
\mathcal{M}_{\text {real }}=\frac{\left|\mathcal{A}_{P+g} \mathcal{A}_{D}\right|^{2}}{\left|\Delta_{X}\right|^{2}}+\frac{\left|\mathcal{A}_{P} \mathcal{A}_{D+g}\right|^{2}}{\left|\Delta_{X g}\right|^{2}}+2 \operatorname{Re}\left[\frac{\left(\mathcal{A}_{P+g} \mathcal{A}_{D}\right)\left(\mathcal{A}_{P} \mathcal{A}_{D+g}\right)^{*}}{\Delta_{X} \Delta_{X g}^{*}}\right]+\ldots
$$

The first two terms can be interpreted as factorizable real corrections to either production or decay of the unstable particle $X$, while the interference term gives rise to non-factorizable 
corrections. Note that the interference term gives a sizeable contribution only if both $\Delta_{X}$ and $\Delta_{X g}$ are resonant, i.e. when the emitted gluon is soft. This shows that, for the real matrix element as well, non factorizable and off-shell effects are encoded by soft radiation. The omitted terms in eq. (2.14), denoted by the ellipses, are suppressed by $\alpha_{s} \delta \sim \delta^{3 / 2}$ compared to the leading tree-level contribution and first contribute to the matrix element at $\mathrm{N}^{3} \mathrm{LO}$ in the power-counting. Hence, they can be neglected in most calculations relevant for hadroncollider phenomenology. However, we point out that they can, in principle, be computed to the desired accuracy in $\delta$, in a way similar to the expansion of the Born amplitude, eq. (2.8).

\subsection{Cross sections}

The real and the virtual amplitudes squared have been split into a hard factorizable part and a soft non-factorizable part. The hard part is further divided into corrections to the production and corrections to the decay. As usual, the virtual and real cross sections, obtained by integrating the squared matrix elements over phase space, have infrared and/or collinear singularities.

The cancellation of these singularities between real and virtual corrections takes place separately for all parts of the cross section. The (hard) production part typically has initial-state collinear singularities that have to be absorbed into the parton distribution functions. It can additionally develop infrared singularities and, when particles other than the unstable particle are present in the final state of the production subprocess, can develop final-state collinear singularities as well. These singularities cancel against the corresponding real singularities of the production part. The (hard) decay part typically has final state collinear singularities and infrared singularities that cancel against the corresponding real singularities.

Finally, the non-factorizable soft corrections only have infrared, but no collinear singularities. Once again, these cancel between the real and virtual corrections. Also, with this cancellation, all explicit scale dependence vanishes and the non-factorizable corrections only depend implicitly on the renormalization and factorization scale. Because the soft emission is governed by a soft scale, $\mu_{s} \sim m_{X} \delta$, it is natural to choose a scale of this order for the coupling from the additional soft emission. The factorization scale and the scale related to the born term are not affected by this and should be kept at the usual hard scale.

\subsection{Mass scheme}

The results given in this section have been expressed in terms of the unstable-particle mass in a generic renormalization scheme, $\hat{m}_{X}$, which is related to the gauge-invariant complex pole of the propagator by $\mu_{X}^{2}=\hat{m}_{X}^{2}+\hat{m}_{X} \Omega_{X}$. While the EFT approach is not limited to a particular scheme, it is more naturally formulated in a class of schemes related to the pole scheme by $\left(\hat{m}_{X}-m_{X}\right) / m_{X} \sim \delta$, which have the property of preserving the EFT scaling $p_{X}^{2}-\hat{m}_{X}^{2} \sim m_{X}^{2} \delta$ under renormalization. The $\overline{\mathrm{MS}}$ is not such a scheme since $\left(\hat{m}_{X, \overline{\mathrm{MS}}}-m_{X}\right) / m_{X, \overline{\mathrm{MS}}} \sim \alpha_{s} \sim \sqrt{\delta}$ and the finite-width expansion becomes less transparent in this case. The pole mass on the other hand, which is the natural mass choice for unstable electroweak gauge bosons and other particles which do not couple to strong interactions, is affected by long-distance ambiguities related to QCD renormalons [24-26] in 
the case of heavy quarks. This makes a precise definition and extraction of this parameter problematic. It is possible to circumvent these issues with the use of so-called "threshold masses" [24, 27-29] or "jet masses" [30,31], which preserve the EFT counting and are not affected by renormalon ambiguities. The implication of the mass-renormalization scheme choice in the case of $t \bar{t}$ production is discussed in section 3.5.

\section{Top-antitop production at hadron colliders}

At hadron colliders the main top-production mechanism is the production of a $t \bar{t}$ pair, which proceeds through strong interactions. ${ }^{3}$ At Born level two partonic processes contribute to the cross section: $q \bar{q} \rightarrow t \bar{t}$, which dominates at Tevatron, and $g g \rightarrow t \bar{t}$, which is the dominant production channel at the LHC. Beyond leading order other partonic production channels contribute as well. Next-to-leading order QCD corrections to the tree-level results, both at the inclusive and differential level, have been known for more than 20 years [32-35] and have been recomputed recently for helicity-specific amplitudes using generalized-unitarity methods [36]. One-loop electroweak contributions are also known [37-41]. At next-to-next-to-leading order in QCD several ingredients and partial results have been known for some time [42-53] and the full NNLO calculations for the fermion-initiated $[54,55]$ and $g q(\bar{q})[56]$ production channels have been recently completed. In addition, resummation of Coulomb singularities and soft logarithms up to next-to-nextto-leading logarithmic accuracy has been studied by several groups [57-61].

All the aforementioned results were computed in the approximation of stable tops. Results in the NWA, including the on-shell decay of the top-antitop pair to the physical final state $W^{+} W^{-} b \bar{b}$, were given in refs. [62-65]. The fully differential semi-leptonic decay of on-shell top quarks is also now known to NNLO [66, 67]. Recently the full NLO QCD calculation of the off-shell top-pair production and decay process in the complex-mass scheme was performed by two separate groups [10-12]. ${ }^{4}$ As expected, finite-width effects were found to be small for the total cross section. However, larger effects were observed for more exclusive distributions, in particular near kinematic boundaries [20]. If such distributions are used for a precise determination of the top-quark mass, the change of shape of distributions caused by finite-width effects has to be under control. This can only be achieved with a careful treatment of finite-width effects in $t \bar{t}$ production.

In this section we present the calculation of the LO and NLO virtual and real amplitudes for $t \bar{t}$ production in the EFT framework, as a non-trivial example of application of the formalism described in section 2. We will focus on the $q \bar{q}$ production channel,

$$
q \bar{q} \rightarrow t \bar{t} \rightarrow W^{+} W^{-} b \bar{b}
$$

\footnotetext{
${ }^{3}$ While the electroweak-mediated production of a single top is numerically only $3-4$ times smaller than top-pair production, a much larger background makes the extraction of the signal in this channel more challenging.

${ }^{4}$ The calculation of ref. [11] treats also the leptonic $W$ decays completely off-shell, while in ref. [10] the $W$ decays are described in narrow-width approximation. The effects of finite $W$-width effects in $t \bar{t}$ production have been investigated recently in ref. [12].
} 
where the decay of the two $W$ bosons to leptons is understood. Our notation and conventions for momentum and colour labelling is given in section 3.1 where we also give explicit expressions for the leading tree-level helicity amplitudes. The calculation of soft and hard virtual corrections as well as the renormalization necessary at NLO are detailed in section 3.3, while the implementation of real corrections is described in section 3.4.

\subsection{Leading tree-level amplitude}

Throughout this work we adopt the following momentum labelling

$$
q\left(p_{1} ; c_{1}\right) \bar{q}\left(p_{2} ; c_{2}\right) \rightarrow b \bar{b} W^{+} W^{-} \rightarrow b\left(p_{3} ; c_{3}\right) \bar{b}\left(p_{4} ; c_{4}\right) \bar{l}_{1}\left(p_{5}\right) \nu_{1}\left(p_{6}\right) l_{2}\left(p_{7}\right) \bar{\nu}_{2}\left(p_{8}\right),
$$

with $c_{1} \ldots c_{4}$ the colour indices of the four external quarks. The leptonic decay of the two $W$-bosons is treated in the NWA. For sake of simplicity, we introduce the shortcuts

$$
\begin{aligned}
& p_{t}=p_{3}+p_{5}+p_{6}, \\
& p_{\bar{t}}=p_{4}+p_{7}+p_{8},
\end{aligned}
$$

and the on-shell projections $\bar{p}_{t}$ and $\bar{p}_{\bar{t}}$, with $\bar{p}_{t}^{2}=\bar{p}_{\bar{t}}^{2}=\mu_{t}^{2}$, which are necessary ${ }^{5}$ for the systematic expansion of the amplitudes in $\delta$. As usual $\mu_{t}^{2}$ denotes the complex pole of the propagator, $\mu_{t}^{2}=m_{t}^{2}-i m_{t} \Gamma_{t}$. We also introduce the abbreviations

$$
\Delta_{t} \equiv p_{t}^{2}-\mu_{t}^{2} \quad \Delta_{\bar{t}} \equiv p_{\bar{t}}^{2}-\mu_{t}^{2},
$$

for the resummed inverse top and antitop propagator. The expression (3.2) is valid in the pole scheme, i.e $m_{t}$ here denotes the pole mass. The conversion to other renormalization schemes is discussed in section 3.5. The amplitudes for the $t \bar{t}$ production process are decomposed onto a basis of colour-state operators, given by

$$
\mathcal{O}_{\{c\}}^{(1)}=\frac{1}{N_{c}} \delta_{c_{1} c_{2}} \delta_{c_{3} c_{4}} \quad \mathcal{O}_{\{c\}}^{(2)}=\frac{2}{\sqrt{N_{c}^{2}-1}} T_{c_{2} c_{1}}^{A} T_{c_{3} c_{4}}^{A},
$$

with $T^{A}$ denoting the generators of the $\mathrm{SU}(3)$ algebra in the fundamental representation and $N_{c}=3$. The two operators describe the $s$-channel exchange of a colour-singlet and colour-octet state respectively, and satisfy the orthonormality condition

$$
\sum_{\{c\}} \mathcal{O}_{\{c\}}^{(i)} \mathcal{O}_{\{c\}}^{(j) *}=\delta_{i j}
$$

At the Born level both doubly-resonant Feynman diagrams containing an intermediate top and antitop pair, as well as singly-resonant and non-resonant diagrams, contribute to the amplitude for the process (3.1). The dominant tree-level contribution to the amplitude is given by the leading term in the expansion in $\delta$ of the left Feynman diagram in figure 5 . In the effective-theory framework, the interaction mediated by the intermediate $s$-channel gluon is replaced by a four-fermion contact interaction (right diagram in figure 5 ) of the form

$$
\frac{i g_{s}^{2}}{\hat{s}} \gamma_{\alpha_{2} \alpha_{1}}^{\mu} \gamma_{\mu, \alpha \bar{\alpha}} T_{c_{2} c_{1}}^{A} T_{c \bar{c}}^{A},
$$

\footnotetext{
${ }^{5}$ Note that in an NLO calculation one can set $\bar{p}_{t}^{2}=\bar{p}_{\bar{t}}^{2}=m_{t}^{2}$ instead, since the term $-i m_{t} \Gamma_{t} \sim m_{t}^{2} \delta$ is formally an NNLO contribution.
} 

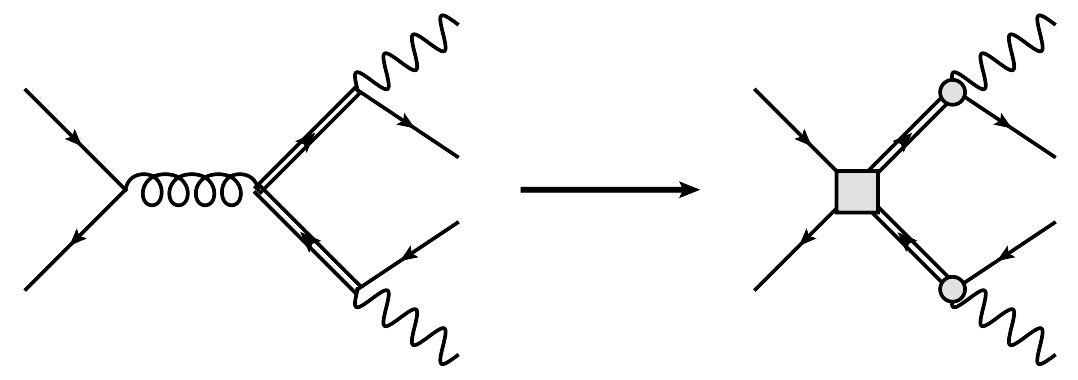

Figure 5. Leading tree-level doubly-resonant contribution to $q \bar{q} \rightarrow b \bar{b} W^{+} W^{-}$in the SM and its EFT counterpart. The grey square and circles represent EFT production and decay matching coefficients.

with $\hat{s}$ the partonic centre-of-mass energy, $\alpha_{1}, \alpha_{2}, \alpha, \bar{\alpha}$ the Lorentz indices of the initial quark and antiquark and final top and antitop, and $c_{1}, c_{2}, c, \bar{c}$ the corresponding colour indices. The tree level amplitude reads (omitting the on-shell decay of the two $W \mathrm{~s}$ )

$$
\mathcal{A}_{\text {tree }}=\frac{\sqrt{N_{c}^{2}-1}}{2} \mathcal{O}_{\{c\}}^{(2)} \mathcal{A}^{(0)},
$$

with

$$
\mathcal{A}^{(0)}=\frac{g_{s}^{2} g^{2}}{2 \hat{s} \Delta_{t} \Delta_{\bar{t}}} \bar{v}\left(\bar{p}_{2}\right) \gamma^{\mu} u\left(\bar{p}_{1}\right) \bar{u}\left(\bar{p}_{3}\right) \notin_{+}^{*}\left(\not_{t}+m_{t}\right) \gamma_{\mu}\left(-\bar{p}_{\bar{t}}+m_{t}\right) \notin_{-}^{*} v\left(\bar{p}_{4}\right)
$$

$\epsilon_{ \pm}$represent the polarization vectors of $W^{ \pm}$. Note that the numerator of $\mathcal{A}^{(0)}$ depends on the projected on-shell momenta which guarantees the gauge-invariance of the result. The contribution of resonant and non-resonant diagrams will be discussed in section 3.2.

Using eq. (2.1) one can easily determine the scaling of the leading amplitude, which is

$$
\mathcal{A}^{(0)} \sim \frac{\alpha_{s} \alpha}{\Delta_{t} \Delta_{\bar{t}}} \sim \delta^{-1 / 2} .
$$

As a consequence of the relative scaling of the top-quark width and the strong coupling constant, $\alpha_{s} \sim \sqrt{\Gamma_{t} / m_{t}}$, the expansion of the amplitude is organized in powers of $\delta^{1 / 2}$. Thus in the following we define as $\mathrm{N}^{k} \mathrm{LO}$ all contributions to the amplitude that scale as $\delta^{(k-1) / 2}$. In particular, NLO corrections, which we will focus on in this paper, scale as $\delta^{0}$. Potentially, these include sub-leading tree-level terms, $\mathcal{O}\left(\alpha_{s}\right)$ hard and soft virtual corrections to the leading doubly-resonant amplitude eq. (3.6), and real QCD corrections. These three contributions will be discussed in turn in the following sections.

\subsection{Sub-leading tree-level contributions}

The first class of corrections which could potentially contribute at NLO in $\delta$ is represented by terms arising from sub-leading tree-level diagrams, which are shown in figure 6 . These include QCD singly-resonant and non-resonant topologies (diagrams (a) and (b)). Since the effective-theory counting of eq. (2.1) relates the expansion in $\alpha_{s}$ to the one in the electroweak coupling constant, electroweak diagrams must also be taken into account beyond LO in $\delta$. For all practical purposes it is sufficient to consider the leading doubly-resonant 


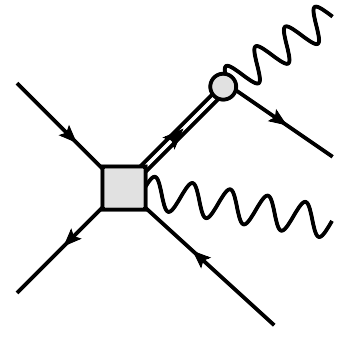

(a)

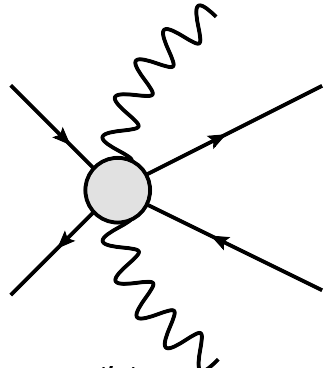

(b)

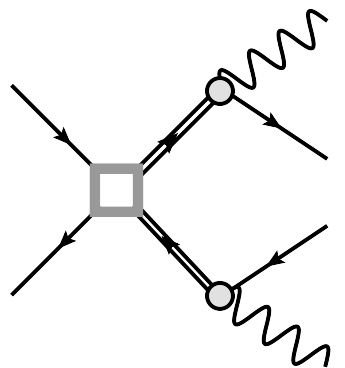

(c)

Figure 6. Subleading tree-level diagrams in the effective theory: (a) singly-resonant diagrams, (b) non-resonant diagrams, (c) doubly-resonant diagrams with electroweak matching coefficients (represented by the grey and white square in the figure). See the text for explanation.

topologies corresponding to diagram (c) in figure 6. Here we use scaling arguments to determine the size of these terms compared to the leading-order amplitude, eq. (3.7).

Contributions with the structure of diagram (a) correspond to the terms on the second line of eq. (2.8), where one of the resonant propagators is either cancelled by higher-order contributions originating from the expansion around the pole, or is not present in the first place. In the effective theory they are described by five-particle contact interactions, whose matching coefficients are proportional to $g_{s}^{2} g_{\text {ew }}$. Their contribution to the amplitude thus scales as

$$
\frac{\alpha_{s} \alpha_{e w}}{\Delta_{t}} \sim \delta^{1 / 2}
$$

which makes them an NNLO correction. Their interference with the leading Born amplitude gives a NNLO correction to the cross section, while the square of diagram (a) contributes to the cross section initially at $\mathrm{N}^{4} \mathrm{LO}$. Non-resonant QCD topologies correspond to six-particle effective interactions (diagram (b)) with matching coefficients starting at order $g_{s}^{2} g_{e w}^{2}$. Since they do not contain intermediate resonant propagators, these scale as

$$
\alpha_{s} \alpha_{e w} \sim \delta^{3 / 2}
$$

and contribute to the cross section as $\mathrm{N}^{4} \mathrm{LO}$ in $\delta$, being suppressed by $\delta^{2}$ compared to the leading Born amplitude.

The doubly resonant electroweak diagrams (diagram (c)) scale as

$$
\frac{\alpha_{e w}^{2}}{\Delta_{t} \Delta_{\bar{t}}} \sim \delta^{0}
$$

Note that in this case the suppression is given by the electroweak nature of the matching coefficients $\left(\sim g_{e w}^{2}\right)$ of the four-particle production operator, rather than by missing resonant propagators. The interference of diagram (c) with the LO amplitude thus gives an NLO correction. However, due to the colour structure of the electroweak production operators, this interference is non-vanishing only for a $b \bar{b}$ initial state. This contribution is numerically suppressed due to the smallness of the bottom-quark PDF inside the proton and is in practice negligible $(<0.1 \%$ of the leading doubly-resonant QCD-mediated diagram). The squared electroweak matrix element counts as an NNLO correction and in this 


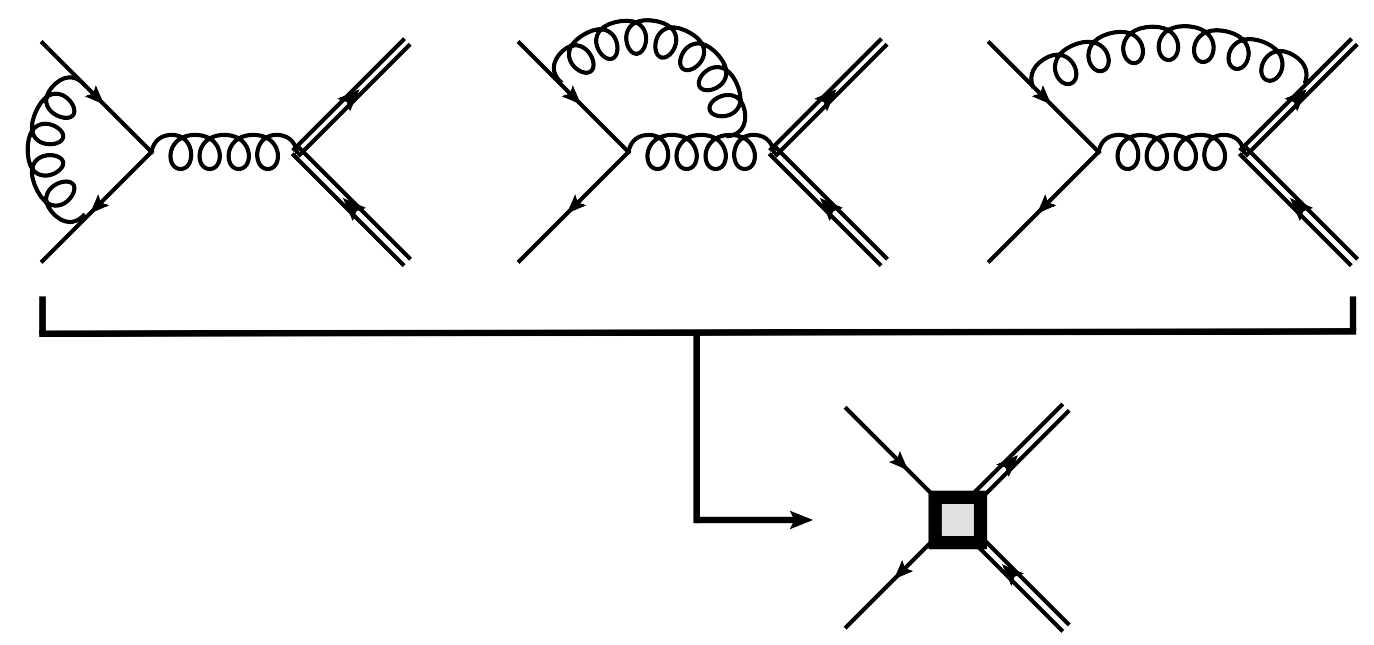

Figure 7. A sample of one-loop diagrams (above) contributing to the $\mathcal{O}\left(\alpha_{s}\right)$ corrections to the production matching coefficient (below).

case there is generally no suppression arising from the parton luminosities, since operators with any light-flavour quark in the initial state contribute.

To conclude, if one neglects the (accidentally) tiny NLO contribution from the interference of diagram (c) with $\mathcal{A}^{(0)}$, the first Born-level corrections to eq. (3.7) arise at NNLO from the interference of singly-resonant QCD diagrams with the leading amplitude and from the squared matrix element of electroweak doubly-resonant diagrams. Being suppressed by $\delta \sim \Gamma_{t} / m_{t}$ compared to eq. (3.7) they are expected to contribute to the cross section at the percent level. This is investigated more quantitatively in section 4 , in particular to test whether the EFT counting applies in practice to the case of $t \bar{t}$ production. As we will see, sub-leading terms account for at most a few percent of the cross section which is in good agreement with the expectation from the EFT scaling arguments.

\subsection{Virtual QCD corrections}

As explained in section 2.2, virtual corrections in the EFT approach are classified into corrections to the hard matching coefficients of the production and decay effective vertices as well as soft corrections to the EFT matrix elements. The former encode physics at the large scale, $q \sim m_{t}$, whilst the latter describe long-scale physics, $q \sim m_{t} \delta$. To obtain NLO accuracy, $\alpha_{s}$ corrections to the hard matching coefficients and one-loop soft corrections are required. As pointed out in section 2.2, $\mathcal{O}\left(\alpha_{s}\right)$ hard matching coefficients can be related to one-loop corrections to the amplitudes for production and decay of on-shell tops. For the production part we use the results of ref. [36], whereas the results for the decay can for example be found in ref. [23]. A sample of the diagrams contributing to the production matching coefficient is shown in figure 7. As for the toy model discussed in section 2.2, hard loops connecting initial and final-state particles, or final-state particles originating from different decays, are suppressed by higher powers of $\delta$. Consider, for example, the two diagrams shown in figure 8. If the momentum flowing in the loop is hard, the intermediate antitop propagator in the first diagram is far off-shell. Thus the diagram is 

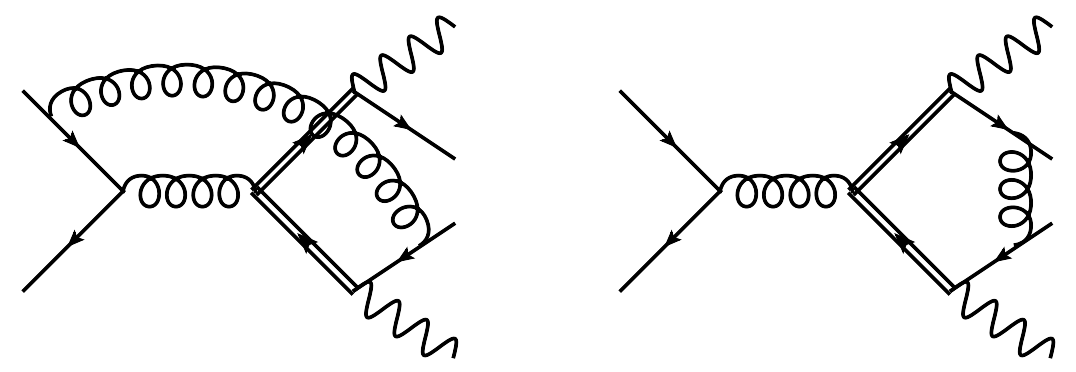

Figure 8. Two examples of one-loop diagrams which do not contribute to the hard matching coefficients at NLO in $\delta$. See the text for further explanation.

suppressed by $\alpha_{s} \Delta_{\bar{t}} \sim \delta^{3 / 2}$ compared to the leading amplitude eq. (3.7), which makes it a $\mathrm{N}^{3} \mathrm{LO}$ correction. The pentagon diagram, in which both top and antitop propagators are off shell, is even more suppressed, $\sim \alpha_{s} \Delta_{t} \Delta_{\bar{t}} \sim \delta^{5 / 2}$, and contributes to the amplitude starting at $\mathrm{N}^{5} \mathrm{LO}$. These contributions do not have to be included in a calculation that aims for NLO accuracy in $\delta$, thus dramatically reducing the computational complexity of the EFT calculation compared to that in the complex-mass scheme.

Contrary to the hard corrections, non-factorizable soft diagrams that connect initial and final states do contribute already at NLO in $\delta$, since the two intermediate top propagators remain resonant if the loop momentum is soft. The complete set of soft one-loop diagrams contributing to the amplitude at order $\delta^{0}$ consists of:

- 2 self-energy corrections, to the top and antitop resonant propagator,

- 8 triangle diagrams, shown in figure 9 ,

- 6 box diagrams, shown in figure 10,

- 1 pentagon diagram, also shown in figure 10 (right bottom corner).

Note that the renormalization of the bare couplings and masses only affects the hard matching coefficients, but does not contribute to the soft corrections. The contribution of the soft diagrams listed above can be obtained by expanding full QCD diagrams in the soft region. Only a consistent expansion in $\delta$ guarantees the gauge invariance of the final result. Most of the integrals shown in figures 9 and 10 were already computed for the case of single-top production $[13,14]$, with the new soft integrals required given by the triangle in figure $9 \mathrm{~d}$, the boxes $10 \mathrm{e}$ and 10f, and the pentagon. As a further example of how the method of regions works we will now briefly discuss the calculation of the triangle diagram 9d.

All soft integrals can be written in terms of the colour operators eq. (3.3), the colourstripped leading-order amplitude eq. (3.7) and scalar functions depending on the invariants $s_{i j} \equiv 2 p_{i} \cdot p_{j}$ built from the external momenta. In particular, the contribution to the amplitude of the triangle in figure $9 \mathrm{~d}$ can be written as

$$
\mathcal{A}_{3, d}^{(1), V}=-\frac{\sqrt{N_{c}^{2}-1}}{4 N_{c}} \mathcal{O}_{\{a\}}^{(2)} \mathcal{A}^{(0)} I_{t \bar{t}}
$$




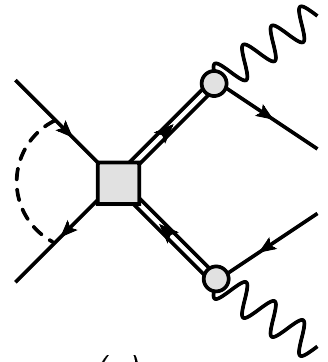

(a)

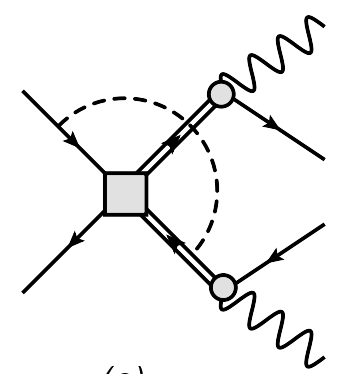

(e)

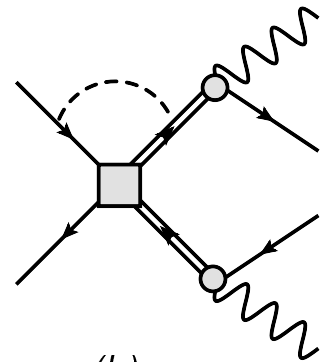

(b)

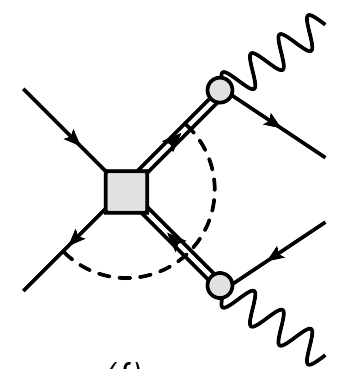

(f)

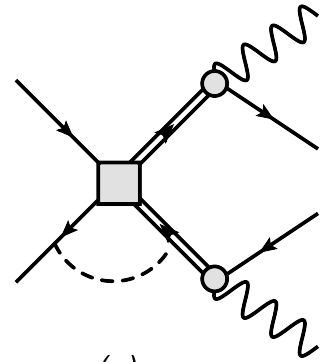

(c)

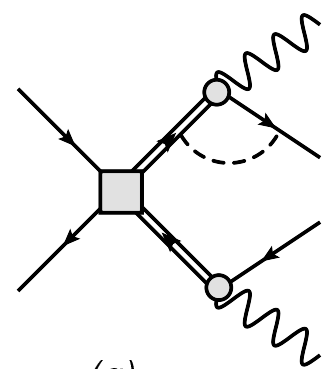

(g)

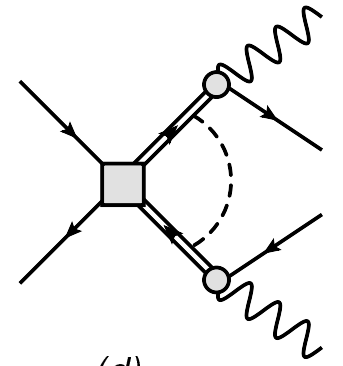

(d)

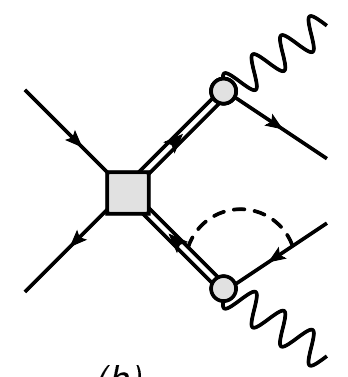

(h)

Figure 9. Soft triangle diagrams contributing at NLO in the EFT counting. Dashed lines represent soft gluons.

with the scalar integral $I_{t \bar{t}}$ defined as

$$
I_{t \bar{t}}=16 \pi i \alpha_{s}\left(\bar{p}_{t} \cdot \bar{p}_{\bar{t}}\right) \frac{e^{\epsilon \gamma_{E}} \mu^{2 \epsilon}}{(4 \pi)^{\epsilon}} \int \frac{d^{d} q}{(2 \pi)^{d}} \frac{1}{q^{2}} \frac{1}{\Delta_{t}-2 \bar{p}_{t} \cdot q} \frac{1}{\Delta_{\bar{t}}+2 \bar{p}_{\bar{t}} \cdot q}
$$

and $d=4-2 \epsilon$. The simple expression of $I_{t \bar{t}}$ follows from the eikonal form of the QCD fermion-gluon interaction vertex in the soft limit, $\bar{u}(p) \gamma^{\mu}\left(\not p_{t} \pm \not q+m_{t}\right) \sim 2 \bar{p}_{t}^{\mu} \bar{u}(p)$, and from the expansion of the propagators in the integrand of the full QCD triangle integral,

$$
\left(p_{t} \pm q\right)^{2}-\mu_{t}^{2}=p_{t}^{2} \pm 2 p_{t} \cdot q+q^{2}-\mu_{t}^{2} \sim \Delta_{t} \pm 2 \bar{p}_{t} \cdot q
$$

where the quadratic term $q^{2} \sim \delta^{2}$ must be dropped, being sub-leading compared to the term $\Delta_{t} \pm 2 \bar{p}_{t} \cdot q \sim \delta$. Note that in eq. (3.13) the off-shell top and antitop momenta $p_{t}$ and $\bar{p}_{t}$ have been replaced by the on-shell projections $\bar{p}_{t}$ and $\bar{p}_{\bar{t}}$, except in the combinations $p_{t}^{2}-\mu_{t}^{2} \equiv \Delta_{t}$ and $\bar{p}_{t}^{2}-\mu_{t}^{2} \equiv \Delta_{\bar{t}}$. In addition, finite-width effects have been resummed into the top propagators, in accordance with the EFT Lagrangian eq. (2.2).

Using Feynman parameters and standard integration techniques eq. (3.13) can be reduced to the one-dimensional integral

$$
I_{t \bar{t}}=\frac{\alpha_{s}}{2 \pi} \eta e^{\epsilon \gamma_{E}} \Gamma(1-\epsilon) \Gamma(2 \epsilon) \int_{0}^{\infty} d y\left[\left(y-\xi_{+}\right)\left(y-\xi_{-}\right)\right]^{-1+\epsilon}\left(\frac{-\Delta_{t}-y \Delta_{\bar{t}}}{m_{t} \mu}\right)^{-2 \epsilon},
$$

where we have introduced the invariant $\eta=2 \bar{p}_{t} \cdot \bar{p}_{\bar{t}} / m_{t}^{2}$ and

$$
\xi_{ \pm}=\frac{\eta \pm \sqrt{\eta^{2}-4+i o_{+}}}{2}
$$




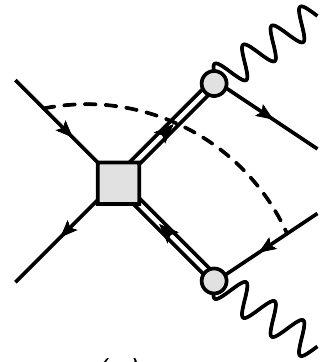

(a)

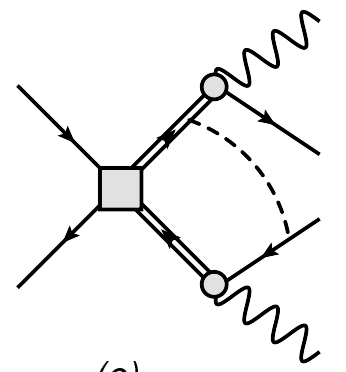

(e)

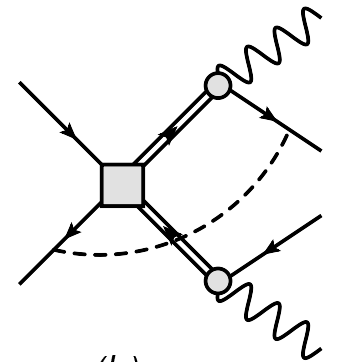

(b)

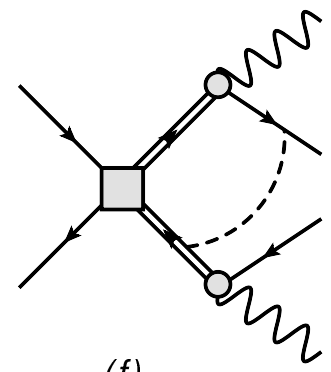

(f)

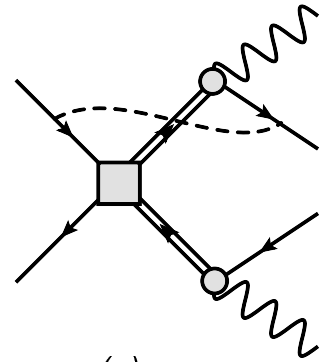

(c)

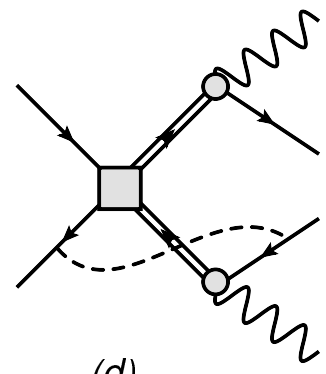

(d)

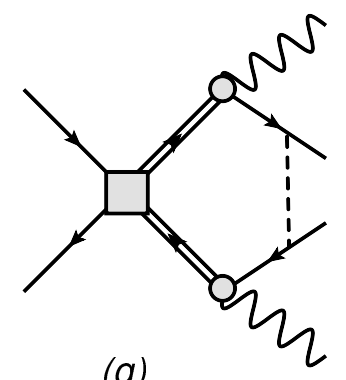

(g)

Figure 10. Soft box and pentagon diagrams contributing at NLO in the EFT counting. Dashed lines represent soft gluons.

with $i o_{+}$an infinitesimal and positive imaginary part. Expanding $I_{t \bar{t}}$ in $\epsilon$ one obtains

$$
I_{t \bar{t}}=\frac{\alpha_{s}}{4 \pi} \eta\left\{I_{t \bar{t}}^{(-1)}\left[\frac{1}{\epsilon}-2 \ln \left(-\frac{\Delta_{t}}{m_{t} \mu}\right)\right]+I_{t \bar{t}}^{(0)}\right\},
$$

where the integrals $I_{t \bar{t}}^{(-1)}$ and $I_{t \bar{t}}^{(0)}$ are given by

$$
\begin{aligned}
I_{t \bar{t}}^{(-1)}= & -\frac{\ln \left(-\xi_{+}\right)-\ln \left(-\xi_{-}\right)}{\xi_{+}-\xi_{-}} \\
I_{t \bar{t}}^{(0)}= & \frac{1}{\xi_{+}-\xi_{-}}\left\{-\frac{1}{2} \ln ^{2}\left(-\xi_{+}\right)+\frac{1}{2} \ln ^{2}\left(-\xi_{-}\right)^{2}-\ln \left(-\xi_{+}\right) \ln \left(\xi_{+}-\xi_{-}\right)\right. \\
& +\ln \left(-\xi_{-}\right) \ln \left(\xi_{-}-\xi_{+}\right)+\frac{1}{2} \ln ^{2}\left(\xi_{+}-\xi_{-}\right)-\frac{1}{2} \ln ^{2}\left(\xi_{-}-\xi_{+}\right) \\
& +\operatorname{Li}_{2}\left(\frac{\xi_{+}}{\xi_{+}-\xi_{-}}\right)-\operatorname{Li}_{2}\left(\frac{\xi_{-}}{\xi_{-}-\xi_{+}}\right)-\ln ^{2}\left(\frac{\Delta_{\bar{t}}}{\Delta_{t}+\Delta_{\bar{t}} \xi_{+}}\right)+\ln ^{2}\left(\frac{\Delta_{\bar{t}}}{\Delta_{t}+\Delta_{\bar{t}} \xi_{-}}\right) \\
& -2 \ln \left(-\xi_{+}\right) \ln \left(\frac{\Delta_{t}}{\Delta_{t}+\Delta_{\bar{t}} \xi_{+}}\right)+2 \ln \left(-\xi_{-}\right) \ln \left(\frac{\Delta_{t}}{\Delta_{t}+\Delta_{\bar{t}} \xi_{-}}\right) \\
& \left.-2 \operatorname{Li}_{2}\left(\frac{\Delta_{\bar{t}} \xi_{+}}{\Delta_{t}+\Delta_{\bar{t}} \xi_{+}}\right)+2 \operatorname{Li}_{2}\left(\frac{\Delta_{\bar{t}} \xi_{-}}{\Delta_{t}+\Delta_{\bar{t}} \xi_{-}}\right)\right\}
\end{aligned}
$$

Note that the only explicit scale dependence in eq. (3.17) is due to the term $\ln \left(-\Delta_{t} /\left(m_{t} \mu\right)\right)$, indicating that the natural scale choice for non-factorizable corrections is $\mu_{\text {soft }} \sim \Delta_{t} / m_{t} \sim$ $\Gamma_{t}$. All other terms in eq. (3.17) have no explicit $\mu$ dependence and do not contain potentially large logarithms. This is a common feature of all soft contributions in figures 9 and 10. The calculation of the missing box and pentagon integrals closely follows the one outlined above for the triangle and will not be given explicitly here. 


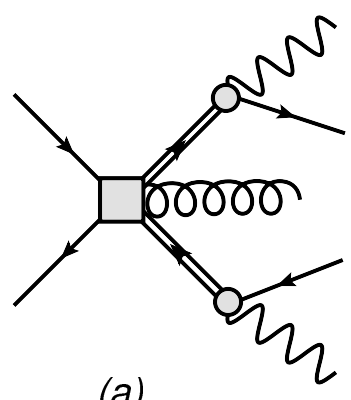

(a)

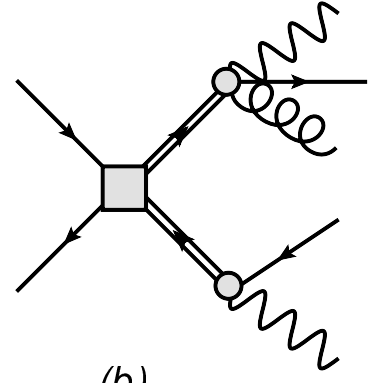

(b)

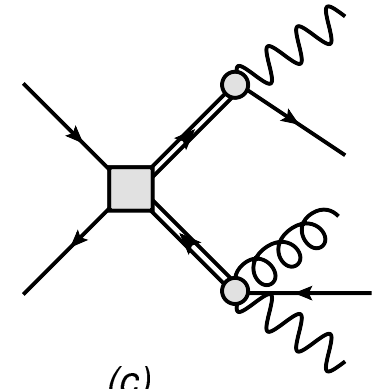

(c)

Figure 11. Real-corrections diagrams contributing to the cross section at NLO in $\delta$.

\subsection{Real QCD corrections}

At NLO real QCD diagrams must also be taken into account. As discussed in section 2.3 and illustrated in figure 11, any real emission diagram can be split into a linear combination of terms containing a single resonant propagator. In turn, these can be interpreted as corrections to either the production or decay subprocesses and their contribution to the amplitude is obtained from the expansion of the full QCD matrix elements around on-shell configurations. These on-shell configurations are defined by $p_{t}^{2}=p_{\bar{t}}^{2}=\mu_{t}^{2}$ for diagram (a), $\left(p_{t}+k\right)^{2}=p_{\bar{t}}^{2}=\mu_{t}^{2}$ for diagram (b) and $p_{t}^{2}=\left(p_{\bar{t}}+k\right)^{2}=\mu_{t}^{2}$ for diagram (c), with $k$ being the momentum of the emitted gluon. Note that here only gluonic corrections to the tree-level topology are considered, but a full calculation which also includes the gluon-initiated production channel would require the calculation of diagrams with a $g q(\bar{q})$ initial state at NLO.

Soft and collinear singularities are treated in the standard way, by adding a suitable subtraction term to the real matrix element and subtracting the corresponding integrated function from the virtual corrections, such that the cancellation of poles is manifest and real corrections can be safely computed in four dimensions. In particular, for the calculation of the results given in section 4, two independent numerical codes were used, one implementing the FKS subtraction method $[68,69]$ and the other, the Catani-Seymour dipole method $[70,71]$. As explained in section 2.4, the cancellation of virtual poles against real ones occurs separately for factorizable and non-factorizable corrections. For factorizable corrections this follows from the cancellation of infrared and collinear singularities for the on-shell production and decay subprocesses. On the other hand, the (infrared) poles of nonfactorizable virtual corrections (i.e. the sum of all soft virtual contributions) are given by

$$
\begin{aligned}
d \sigma_{V, \text { non-fact. }}= & \frac{\alpha_{s}}{6 \pi \epsilon}\left[16+7 \ln \left(\frac{s_{13}}{m_{t}^{2}}\right)+7 \ln \left(\frac{s_{24}}{m_{t}^{2}}\right)+2 \ln \left(\frac{s_{14}}{m_{t}^{2}}\right)+2 \ln \left(\frac{s_{23}}{m_{t}^{2}}\right)\right. \\
& -\ln \left(\frac{s_{34}}{m_{t}^{2}}\right)-7 \ln \left(\frac{s_{1 t}}{m_{t}^{2}}\right)-7 \ln \left(\frac{s_{2 \bar{t}}}{m_{t}^{2}}\right)-2 \ln \left(\frac{s_{1 \bar{t}}}{m_{t}^{2}}\right)-2 \ln \left(\frac{s_{2 t}}{m_{t}^{2}}\right) \\
& \left.-8 \ln \left(\frac{s_{3 t}}{m_{t}^{2}}\right)-8 \ln \left(\frac{s_{4 \bar{t}}}{m_{t}^{2}}\right)+\frac{\eta\left[\ln \left(-\xi_{+}\right)-\ln \left(-\xi_{-}\right)\right]}{2\left(\xi_{+}-\xi_{-}\right)}\right] d \sigma_{\text {Born }},
\end{aligned}
$$

with $s_{i j}=2 p_{i} \cdot p_{j}$. These can be shown to coincide with the pole structure of the productiondecay interference terms in eq. (2.14). 


\subsection{Mass-scheme choice}

The results shown in the previous sections have been computed in the so-called "pole-mass scheme", in which the renormalized mass parameter is chosen to coincide with the real part of the complex pole of the top-quark propagator. This particular mass scheme is affected by ambiguities related to QCD renormalons [24-26], making a determination of the top-quark mass at an accuracy better than $\Lambda_{\mathrm{QCD}}$ conceptually impossible. Avoiding these problems requires the use of a mass scheme which is not affected by long-distance ambiguities. An example of such a mass is the $\overline{\mathrm{MS}}$-renormalized mass, $m_{t}^{\overline{\mathrm{MS}}}$. On the other hand the $\overline{\mathrm{MS}}$ mass presents the unpleasant feature of not preserving the effective-theory counting (or, in other words, it differs from the pole mass by terms of order $\alpha_{s}$ ), thus spoiling the EFT counting scheme. More suitable choices are represented by the so-called "threshold masses" [24, 2729], introduced in the context of heavy-quark threshold physics, and "jet masses" [30, 31] studied for boosted top-jet events in $e^{+} e^{-}$-collisions. These masses have the attractive feature of not being sensitive to low-momentum physics and of differing only by amounts of order $\sim m_{t} \alpha_{s}^{2} \sim m_{t} \delta$ from the pole mass, thus preserving the effective-theory counting. In the following we will consider the "potential-subtracted" (PS) mass definition [27] as an alternative suitable mass scheme, illustrating how this fits into the effective-theory calculation.

The relation between the pole mass and the PS mass can be found in ref. [27], and up to second order in $\alpha_{s}$ (enough for applications in this work) reads

$$
m_{t}=m_{t, \mathrm{PS}}+\mu_{\mathrm{PS}}\left[\frac{\alpha_{s}}{2 \pi} \delta_{1}^{\mathrm{PS}}+\left(\frac{\alpha_{s}}{2 \pi}\right)^{2} \delta_{2}^{\mathrm{PS}}\right],
$$

with

$$
\begin{aligned}
& \delta_{1}^{\mathrm{PS}}=2 C_{F} \\
& \delta_{2}^{\mathrm{PS}}=C_{F}\left[a_{1}-b_{0}\left(\ln \left(\frac{\mu_{\mathrm{PS}}^{2}}{\mu_{R}^{2}}\right)-2\right)\right]
\end{aligned}
$$

and $a_{1}=31 / 3-10 n_{f} / 9, b_{0}=11-2 n_{f} / 3$. The cutoff $\mu_{\mathrm{PS}}$ has to be chosen of order $\sim m_{t} \alpha_{s}$ to preserve the EFT counting. Substituting the pole mass in favour of the PS mass in the (renormalized) resummed top propagator one obtains

$$
\frac{1}{p_{t}^{2}-m_{t, \mathrm{PS}}^{2}+i m_{t, \mathrm{PS}} \Gamma_{t}-\frac{\alpha_{s}}{\pi} \delta_{1}^{\mathrm{PS}} \mu_{\mathrm{PS}} m_{t, \mathrm{PS}}-\frac{\alpha_{s}^{2}}{2 \pi^{2}} \delta_{2}^{\mathrm{PS}} \mu_{\mathrm{PS}} m_{t, \mathrm{PS}}+\ldots}
$$

with the ellipses denoting terms that scale as $\delta^{2}$ or higher. Note that, counting $\mu_{\mathrm{PS}} \sim \sqrt{\delta}$, the term $-\alpha_{s} \delta_{1}^{\mathrm{PS}} \mu_{\mathrm{PS}} m_{t, \mathrm{PS}} / \pi$ scales as the leading term $p_{t}^{2}-m_{t, \mathrm{PS}}^{2}+i m_{t, \mathrm{PS}} \Gamma_{t} \sim \delta$, and has to be kept in the denominator of the propagator. The term proportional to $\alpha_{s}^{2}$ is suppressed by an additional $\sqrt{\delta}$ and can be re-expanded to fixed-order in the expansion parameters,

$$
\frac{1}{p_{t}^{2}-m_{t, \mathrm{PS}}^{2}+i m_{t, \mathrm{PS}} \Gamma_{t}-\frac{\alpha_{s}}{\pi} \delta_{1}^{\mathrm{PS}} \mu_{\mathrm{PS}} m_{t, \mathrm{PS}}}\left\{1+\frac{\frac{\alpha_{s}^{2}}{2 \pi^{2}} \delta_{2}^{\mathrm{PS}} \mu_{\mathrm{PS}} m_{t, \mathrm{PS}}}{p_{t}^{2}-m_{t, \mathrm{PS}}^{2}+i m_{t, \mathrm{PS}} \Gamma_{t}-\frac{\alpha_{s}}{\pi} \delta_{1}^{\mathrm{PS}} \mu_{\mathrm{PS}} m_{t, \mathrm{PS}}}\right\} .
$$


Note that the replacement $m_{t} \rightarrow m_{t, \mathrm{PS}}+\frac{\alpha_{s}}{2 \pi} \delta_{1}^{\mathrm{PS}} \mu_{\mathrm{PS}}+\ldots$ in the numerator of the matrix elements does not generate extra terms at NLO since $\alpha_{s} \mu_{\mathrm{PS}} \sim \delta$. Thus to convert the polescheme result for the renormalized cross section to the PS scheme it is sufficient to replace $m_{t}^{2}-i m_{t} \Gamma_{t} \rightarrow m_{t, \mathrm{PS}}^{2}-i m_{t, \mathrm{PS}} \Gamma_{t}+\frac{\alpha_{s}}{\pi} \delta_{1}^{\mathrm{PS}} \mu_{\mathrm{PS}} m_{t, \mathrm{PS}}$ in the denominator of the top (antitop) propagator (in both the LO and NLO cross section), $m_{t} \rightarrow m_{t, \mathrm{PS}}$ in the numerator and to add the higher-order propagator contribution

$$
\delta \mathcal{A}_{\mathrm{pole} \rightarrow \mathrm{PS}}^{(1)}=\mathcal{A}^{(0)} \frac{\frac{\alpha_{s}^{2}}{2 \pi^{2}} \delta_{2}^{\mathrm{PS}} \mu_{\mathrm{PS}} m_{t, \mathrm{PS}}}{p_{t}^{2}-m_{t, \mathrm{PS}}^{2}+i m_{t, \mathrm{PS}} \Gamma_{t}-\frac{\alpha_{s}}{\pi} \delta_{1}^{\mathrm{PS}} \mu_{\mathrm{PS}} m_{t, \mathrm{PS}}}
$$

to the NLO amplitude and a similar one for the internal antitop propagator.

The result for the conversion from pole scheme to PS scheme can be equivalently obtained considering how the top-quark propagator is renormalized and resummed in the two schemes. We introduce the short-cut $D_{t}=p_{t}^{2}-m_{t}^{2}$ and $D_{t, \mathrm{PS}}=p_{t}^{2}-m_{t, \mathrm{PS}}^{2}$ to indicate the renormalized inverse propagator in the pole and PS scheme (without selfenergy resummation). In the pole scheme, the contribution of the hard part of the one-loop QCD self-energy to the internal top-quark line can be written as [13]

$$
\delta_{\mathrm{QCD}}\left(m_{t}\right)\left[\frac{2 i m_{t}^{2}\left(\not p_{t}+m_{t}\right)}{D_{t}^{2}}+\frac{i m_{t}}{D_{t}}-\frac{i\left(\not p_{t}+m_{t}\right)}{D_{t}}\right]
$$

with

$$
\delta_{\mathrm{QCD}}\left(m_{t}\right)=\frac{\alpha_{s} C_{F}}{2 \pi}\left[\frac{3}{2 \epsilon}+2+\frac{x_{s c}}{2}-\frac{3}{2} \ln \left(\frac{m_{t}^{2}}{\mu^{2}}\right)\right]+\mathcal{O}\left(\alpha_{s}^{2}\right),
$$

while the correction to the top-quark line from the mass-renormalization counterterm $\delta m_{t}$ reads

$$
\frac{\delta m_{t}}{m_{t}}\left[\frac{2 i m_{t}^{2}\left(\not p_{t}+m_{t}\right)}{D_{t}^{2}}+\frac{i m_{t}}{D_{t}}\right] .
$$

In the pole scheme one has $\delta m_{t} / m_{t}=-\delta_{\mathrm{QCD}}\left(m_{t}\right)$, which leads to the renormalized one-loop propagator

$$
\frac{i\left(\not p_{t}+m_{t}\right)}{D_{t}}\left(1-\delta_{\mathrm{QCD}}\left(m_{t}\right)\right)
$$

Including in addition the hard part of the electroweak self energy one obtains

$$
\frac{i\left(\not p_{t}+m_{t}\right)}{\Delta_{t}}\left(1-\delta_{\mathrm{QCD}}\left(m_{t}\right)\right) \text {. }
$$

In the PS scheme the self-energy correction to the propagator has the same functional form as in the pole scheme, but it depends on the PS mass $m_{t, \mathrm{PS}}$

$$
\delta_{\mathrm{QCD}}\left(m_{t, \mathrm{PS}}\right)\left[\frac{2 i m_{t, \mathrm{PS}}^{2}\left(\not p_{t}+m_{t, \mathrm{PS}}\right)}{D_{t, \mathrm{PS}}^{2}}+\frac{i m_{t, \mathrm{PS}}}{D_{t, \mathrm{PS}}}-\frac{i\left(\not t_{t}+m_{t, \mathrm{PS}}\right)}{D_{t, \mathrm{PS}}}\right] .
$$

Analogously, the mass renormalization gives the contribution

$$
\frac{\delta m_{t, \mathrm{PS}}}{m_{t, \mathrm{PS}}}\left[\frac{2 i m_{t, \mathrm{PS}}^{2}\left(\not p_{t}+m_{t, \mathrm{PS}}\right)}{D_{t, \mathrm{PS}}^{2}}+\frac{i m_{t, \mathrm{PS}}}{D_{t, \mathrm{PS}}}\right] .
$$


The mass counterterm in the PS scheme can be obtained using the relation between the pole and PS mass, eq. (3.21), and the results given in eqs. (3.20) and (3.24) of ref. [13]

$$
\frac{\delta m_{t, \mathrm{PS}}}{m_{t, \mathrm{PS}}}=-\delta_{\mathrm{QCD}}\left(m_{t, \mathrm{PS}}\right)+\frac{\mu_{\mathrm{PS}}}{m_{t, \mathrm{PS}}}\left[\frac{\alpha_{s}}{2 \pi} \delta_{1}^{\mathrm{PS}}+\left(\frac{\alpha_{s}}{2 \pi}\right)^{2} \delta_{2}^{\mathrm{PS}}+\ldots\right] .
$$

Thus the one-loop renormalized propagator in the PS scheme reads

$$
\begin{aligned}
& \frac{i\left(\not p_{t}+m_{t, \mathrm{PS}}\right)}{D_{t, \mathrm{PS}}}\left(1-\delta_{\mathrm{QCD}}\left(m_{t, \mathrm{PS}}\right)\right) \\
& +\frac{\mu_{\mathrm{PS}}}{m_{t, \mathrm{PS}}}\left[\frac{\alpha_{s}}{2 \pi} \delta_{1}^{\mathrm{PS}}+\left(\frac{\alpha_{s}}{2 \pi}\right)^{2} \delta_{2}^{\mathrm{PS}}\right]\left(\frac{2 i m_{t, \mathrm{PS}}^{2}\left(\not t_{t}+m_{t, \mathrm{PS}}\right)}{D_{t, \mathrm{PS}}^{2}}+\frac{i m_{t, \mathrm{PS}}}{D_{t, \mathrm{PS}}}\right) .
\end{aligned}
$$

Remembering that $\mu_{\mathrm{PS}} \sim \alpha_{s} m_{t}$ and keeping only the parametrically relevant terms one obtains

$$
\frac{i\left(\not p_{t}+m_{t, \mathrm{PS}}\right)}{D_{t, \mathrm{PS}}}\left(1-\delta_{\mathrm{QCD}}\left(m_{t, \mathrm{PS}}\right)+\frac{\frac{\alpha_{s}}{\pi} \delta_{1}^{\mathrm{PS}} \mu_{\mathrm{PS}} m_{t, \mathrm{PS}}+\frac{\alpha_{s}^{2}}{2 \pi^{2}} \delta_{2}^{\mathrm{PS}} \mu_{\mathrm{PS}} m_{t, \mathrm{PS}}}{D_{t, \mathrm{PS}}}\right) .
$$

The terms proportional to $\delta_{\mathrm{QCD}}$ and $\delta_{2}^{\mathrm{PS}}$ scale as $\sim \alpha_{s} \sim \sqrt{\delta}$ compared to the leading propagator, and can be included perturbatively in the calculation of the cross section. In contrast, the term proportional to $\delta_{1}^{\mathrm{PS}}$ is a correction of order one, since $\alpha_{s} \mu_{\mathrm{PS}} m_{t, \mathrm{PS}} / D_{t, \mathrm{PS}} \sim 1$. It thus has to be resummed into the leading-order propagator. Including the contribution of the one-loop electroweak self energy, the resummed renormalized propagator reads

$$
\frac{i\left(\not p_{t}+m_{t, \mathrm{PS}}\right)}{\Delta_{t, \mathrm{PS}}-\frac{\alpha_{s}}{\pi} \delta_{1}^{\mathrm{PS}} \mu_{\mathrm{PS}} m_{t, \mathrm{PS}}}\left[1-\delta_{\mathrm{QCD}}\left(m_{t, \mathrm{PS}}\right)+\frac{\frac{\alpha_{s}^{2}}{2 \pi^{2}} \delta_{2}^{\mathrm{PS}} \mu_{\mathrm{PS}} m_{t, \mathrm{PS}}}{\Delta_{t, \mathrm{PS}}-\frac{\alpha_{s}}{\pi} \delta_{1}^{\mathrm{PS}} \mu_{\mathrm{PS}} m_{t, \mathrm{PS}}}\right],
$$

with $\Delta_{t, \mathrm{PS}}=p_{t}^{2}-m_{t, \mathrm{PS}}^{2}+i m_{t, \mathrm{PS}} \Gamma_{t}$. This confirms that the conversion from pole to PS scheme amounts to the replacement $\Delta_{t} \rightarrow \Delta_{t, \mathrm{PS}}-\frac{\alpha_{s}}{\pi} \delta_{1}^{\mathrm{PS}} \mu_{\mathrm{PS}} m_{t, \mathrm{PS}}$ in the denominator of the resummed top-quark propagator and the addition of the term

$$
\delta \mathcal{A}_{\mathrm{pole} \rightarrow \mathrm{PS}}^{(1)}=\mathcal{A}^{(0)} \frac{\frac{\alpha_{s}^{2}}{2 \pi^{2}} \delta_{2}^{\mathrm{PS}} \mu_{\mathrm{PS}} m_{t, \mathrm{PS}}}{\Delta_{t, \mathrm{PS}}-\frac{\alpha_{s}}{\pi} \delta_{1}^{\mathrm{PS}} \mu_{\mathrm{PS}} m_{t, \mathrm{PS}}}
$$

to the NLO amplitude for each intermediate top or antitop quark line present at LO.

\subsection{Validity of EFT results}

We conclude this section with a remark on the validity of the EFT results for $t \bar{t}$ production. As outlined in section 2, in our effective-theory framework the unstable particle is correctly treated by HQET (plus finite-width effects). Strictly speaking, for production of a pair of unstable particles this is true as long as the two velocities $v, \bar{v}$ are relativistic, which is the case if $\sqrt{\hat{s}} \gg 2 m_{t}$, namely when the partonic centre-of-mass energy $\sqrt{\hat{s}}$ is much larger than the pair-production threshold. When $\sqrt{\hat{s}}-2 m_{t} \sim \Gamma_{t}$ the two particles become nonrelativistic, $v \sim \bar{v} \sim(1, \overrightarrow{0})$, and the correct effective theory to describe the pair-production process is non-relativistic QCD (NRQCD). In the expansion by regions this is seen as an 
additional potential region which develops when the heavy particles become non-relativistic, and describes Coulomb interactions of the two slow-moving particles. This breakdown of the expansion in $\delta$ was already noticed in the context of the pole approximation for $W$ pair production, with the double-pole approximation quickly loosing accuracy when $\sqrt{\hat{s}}$ approaches $2 M_{W}$ [72]. While this is relevant for an $e^{-} e^{+}$collider, where the centre-ofmass energy can be tuned to probe the threshold region, at hadron colliders the physical hadronic cross section is obtained by convoluting the partonic cross section and parton luminosities over the full range $\sqrt{\hat{s}}=[0, \sqrt{s}]$. For a multi-TeV hadronic c.o.m. energy $\sqrt{s}$, and particle masses in the range of $100-200 \mathrm{GeV}$, the contribution of the threshold region is expected to be small, so that the error introduced by using the wrong EFT description near threshold is negligible. We have checked this explicitly for the total NLO cross section in the stable-top approximation and found that the threshold region, defined by $v \leq 0.1-0.2$, contributes $1-5 \%$ of the total hadronic cross section. The Coulomb terms, which are the ones incorrectly reproduced by HQET in the threshold limit, are in fact less than $1 \%$.

Below threshold, doubly-resonant configurations do not dominate the cross section because the phase space for production of two resonant tops is strongly suppressed for $\sqrt{\hat{s}}<2 m_{t}$. In this region NRQCD should be replaced by a new effective theory describing singly-resonant production of a $t \bar{t}^{*}\left(t^{*} \bar{t}\right)$ pair, which would require the calculation of non-trivial QCD corrections to the processes $q \bar{q} \rightarrow t W^{-} \bar{b}\left(\bar{t} W^{+} b\right)$. For this reason, below threshold we switch to a Born-level prediction of the top-pair production cross section, and set virtual and real corrections to zero. Once again, one expects the error in introducing this approximation to be below the accuracy of a few percent which we pursue here. Actually, in the results presented next, we impose a physical cut on the final state so that we never run into the complications near threshold.

\section{Results}

In this section we present explicit results for a sample of representative kinematical distributions relevant for top quark pair production studies. Our main purpose here is to discuss the importance of off-shell and factorizable corrections by making a direct comparison to the NWA approach. The distributions presented in sections 4.1-4.3 are computed in the pole-mass renormalization scheme whilst a comparison to results obtained in the PS scheme is given in section 4.4 .

The input values for the SM parameters are given in table 1. For the convolution of the partonic cross section with the PDFs we use the MSTW2008 NLO set [73] for both LO and NLO cross sections, taking the corresponding value for $\alpha_{s}$ for consistency. In addition, for all contributions involving the top decay width we use the NLO width, $\Gamma_{t}^{\mathrm{NLO}}$. Using NLO PDFs and $\Gamma_{t}^{\mathrm{NLO}}$ throughout is a choice that has been made to ease identification of off-shell effects, ensuring that corrections arising from changes in PDFs or decay widths do not cloud the effects introduced by the off-shellness of the tops. Our default choice of factorization and renormalization scales is $\mu_{F}=\mu_{R}=m_{t}$. Where present, bands around LO and NLO off-shell results are obtained by simultaneously varying the renormalization and factorization scales in the interval $\left[m_{t} / 2,2 m_{t}\right]$. 


\begin{tabular}{|ccc|}
\hline \multicolumn{3}{|c|}{ Collider: Tevatron, $\sqrt{s}=1.96 \mathrm{TeV}$} \\
\hline$p_{T}\left(J_{b}\right)>15 \mathrm{GeV}$ & $p_{T}\left(l^{+}\right)>15 \mathrm{GeV}$ & $\mathscr{E}_{T}>20 \mathrm{GeV}$ \\
$p_{T}\left(J_{\bar{b}}\right)>15 \mathrm{GeV}$ & $p_{T}\left(l^{-}\right)>15 \mathrm{GeV}$ & $D_{\text {jet }}=0.7$ \\
\hline$M_{t}=172.9 \mathrm{GeV}$ & $\Gamma_{t}^{\mathrm{NLO}}=1.3662 \mathrm{GeV}$ & $M_{Z}=91.2 \mathrm{GeV}$ \\
$M_{W}=80.4 \mathrm{GeV}$ & $\Gamma_{W}=2.14 \mathrm{GeV}$ & $\alpha_{\mathrm{ew}}=0.03394$ \\
\hline
\end{tabular}

Table 1. Example process definition at the Tevatron collider and parameter setup.

Throughout the following discussion we assume that the final state contains a $b$ and a $\bar{b}$ jet and furthermore that the $W$-bosons are perfectly reconstructed. Despite the latter not being feasible in the di-lepton channel it allows one to identify features arising from the off-shellness of the top quarks that will also be relevant in an experimentally rigorous analysis. It is emphasised however, that wherever we discuss an observable related to the (anti)top, it is understood this is the reconstructed (anti)top quark, defined in terms of the $W^{+(-)}$-boson and $(\bar{b}) b$-jet, $J_{b(\bar{b})}$.

In order that the EFT approach and counting remains valid the following conditions on the reconstructed top-quark momenta, $p(t)=p\left(J_{b}\right)+p\left(W^{+}\right)$and $p(\bar{t})=p\left(J_{\bar{b}}\right)+p\left(W^{-}\right)$, are also imposed:

$$
\begin{aligned}
140 \mathrm{GeV}<M_{\text {inv }}(t) & =\sqrt{(p(t))^{2}}<200 \mathrm{GeV}, \\
140 \mathrm{GeV}<M_{\text {inv }}(\bar{t}) & =\sqrt{(p(\bar{t}))^{2}}<200 \mathrm{GeV}, \\
M_{\text {inv }}(t \bar{t}) & =\sqrt{(p(t)+p(\bar{t}))^{2}}>350 \mathrm{GeV} .
\end{aligned}
$$

The experimental setup we consider, typical for a study of top-pair production, is detailed in table 1 and defines the process

$$
p \bar{p} \rightarrow J_{b} J_{\bar{b}} \notin_{T} l^{+} l^{-}+X .
$$

We note that the jets have been clustered using the standard $k_{t}$-algorithm with jet resolution parameter $D_{j e t}=0.7$.

The implementation of the factorizable corrections to production and decay subprocesses has been checked in the limit of on-shell top quarks against the di-lepton channel implementation in MCFM [65]. Very good agreement was found both for inclusive cross sections and for all distributions we have checked. The analytical expressions for the virtual non-factorizable corrections have been put through extensive numerical checks and corresponding real corrections display the correct independence on the soft FKS parameter $\xi_{\text {cut }}$.

\subsection{Invariant and transverse masses}

We start by presenting results for the invariant and transverse masses of the reconstructed tops, displayed in figures 12 and 13 respectively. These observables are expected to be 
sensitive to the off-shellness of the top quarks. The off-shell LO ( $\sigma^{\mathrm{LO} \text {, off-s. }}$, green band) and NLO $\left(\sigma^{\mathrm{NLO} \text {, off-s. }}\right.$, red band) results are displayed in the upper plots, as is the NLO prediction in the NWA $\left(\sigma^{\mathrm{NLO}, \text { on-s. }}\right.$, blue, solid). Note that even though the top quarks are strictly on-shell in the NWA, beyond LO the invariant mass of the reconstructed top (i.e. the $W^{+} J_{b}$ system) is not necessarily equal to $m_{t}$. In order to straightforwardly assess the importance of off-shell effects, non-factorizable corrections $\left(\sigma^{\mathrm{NF}}\right.$, interference between radiation from production and decay) and sub-leading contributions $\left(\sigma^{\text {sub-lead. }}\right.$, non-resonant tree-level contributions), we study the quantities

$$
\begin{array}{ll}
\frac{\sigma^{\mathrm{NLO}, \text { off-s. }}-\sigma^{\mathrm{NLO}, \text { on-s. }}}{\sigma^{\mathrm{NLO}, \text { on-s. }}} & \text { (red, solid), } \\
\frac{\sigma^{\mathrm{NF}}}{\sigma^{\mathrm{NLO}, \text { on-s. }}} & \text { (blue, solid), } \\
\frac{\sigma^{\text {sub-lead. }}}{\sigma^{\mathrm{NLO}, \text { on-s. }}} & \text { (green, dashed), }
\end{array}
$$

in the lower panel of each figure. The non-factorizable corrections have been evaluated at the scale $\mu_{\text {soft }} \simeq m_{t} \delta$, as motivated at the end of section 3.3. For the case at hand this amounts to multiplying $\sigma^{\mathrm{NF}}\left(\mu=m_{t}\right)$ by the factor $\alpha_{s}\left(\mu_{\mathrm{soft}}\right) / \alpha_{s}\left(m_{t}\right) \simeq 2.24$, when $\delta=0.02$. It would also be desirable to include a resummation of logarithms of $\mu_{\text {soft }} / m_{t}$ by running the hard matching coefficients down to the soft scale using RGEs, however this will not be discussed here.

Firstly we focus on the invariant mass distribution of the reconstructed antitop quark, shown in figure $12 .{ }^{6}$ An important feature present in the upper plot is that the shapes of the NLO off-shell and on-shell distributions are significantly different, particularly near and beyond the peak position. This is made clear in the lower inset where one observes that off-shell effects are indeed sizeable, exceeding $60 \%$ around the peak. However, one also notices that they change sign in this region, resulting in the net off-shell effects being small due to an averaging out that occurs for inclusive enough observables like the total cross section. The lower inset in figure 12 also shows the effect of strict non-factorizable effects and sub-leading contributions, both normalized to the NLO on-shell results. Nonfactorizable corrections, given by the sum of virtual and real soft contributions, are very small, $\sim 1 \%$ over the whole invariant-mass range considered, except around the pole, where they grow to roughly $6-7 \%$. The contribution of sub-leading diagrams is also very small close to the peak, where $\delta \ll 1$ and the effective-theory counting is satisfied, but becomes sizeable in the tail region for $M_{\text {inv }}(t)>m_{t}$, as one would expect.

We now consider figure 13 which displays the transverse mass distribution of the reconstructed top quark, defined via

$$
M_{T}(t)=\left(\left(\left|\vec{p}_{T}\left(J_{b}\right)\right|+\left|\vec{p}_{T}\left(l^{+}\right)\right|+\left|E_{T}\left(\nu_{l}\right)\right|\right)^{2}-\left(\vec{p}_{T}\left(J_{b}\right)+\vec{p}_{T}\left(l^{+}\right)+\vec{p}_{T}\left(\nu_{l}\right)\right)^{2}\right)^{1 / 2}
$$

where $\vec{p}_{T}(k)$ is the transverse momentum of the final state $k$.

Once more, it is clear that there are substantial differences between the shape of the distribution in the on-shell and off-shell approaches. In particular, the relatively sharp

\footnotetext{
${ }^{6}$ The LO on-shell result is a delta function centred at $m_{t}$ and it is omitted in the plot.
} 


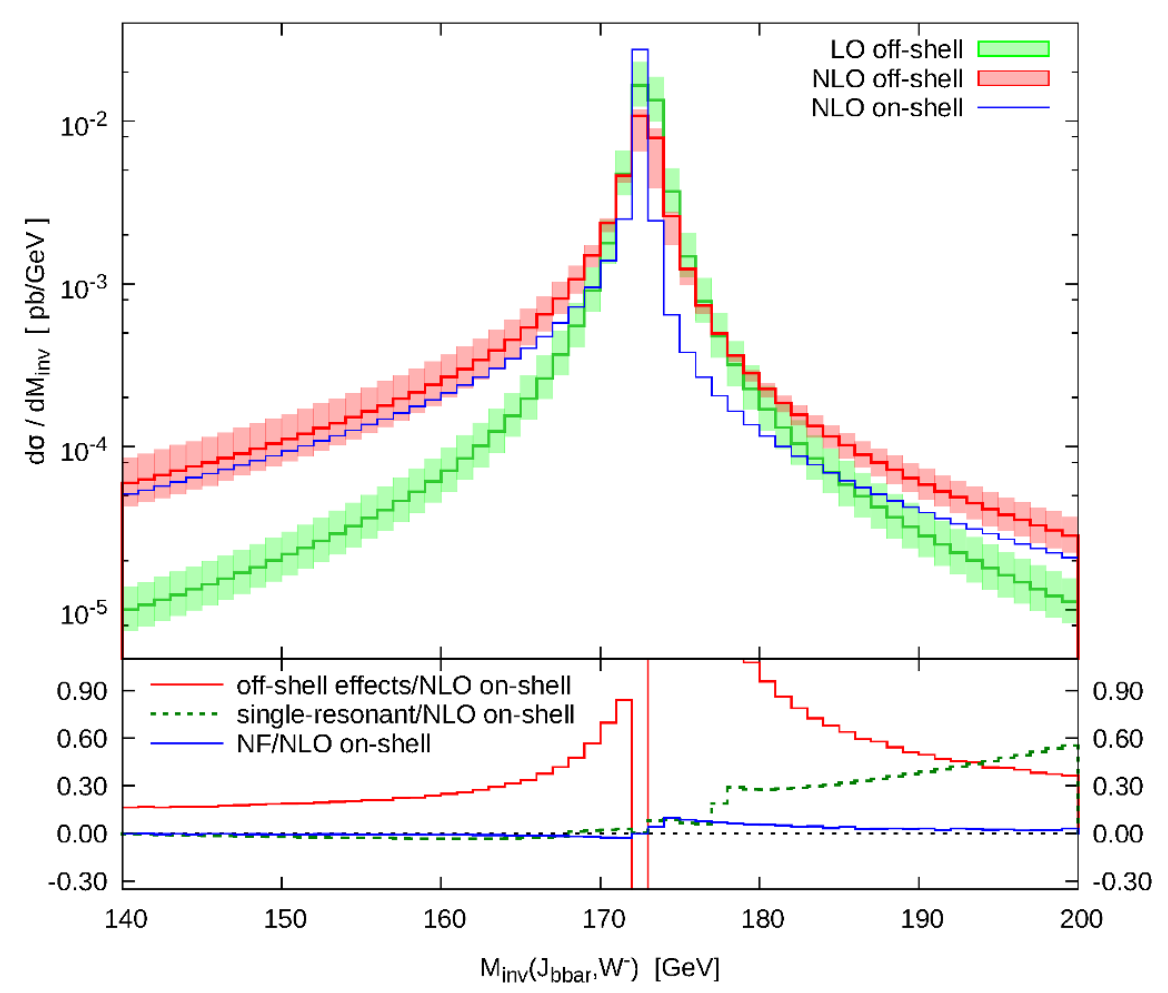

Figure 12. Invariant mass distribution for the reconstructed antitop quark. Upper plot: results for the off-shell LO result (green), off-shell NLO result (red) and on-shell NLO prediction (blue). Lower inset: relative contributions of total off-shell effects, non-factorizable corrections and singly-resonant diagrams. See the text for explanation.

edge in the on-shell calculation becomes much less steep once the on-shell assumption is relaxed. The size of off-shell effects are quantified in the lower inset where one observes that these constitute a negative 2-3\% correction to the NWA result, except near and beyond the edge (at $M_{T} \sim m_{t}$ ) where much larger effects are present. In this region, the averaging effect mentioned earlier is less effective leading to enhanced corrections. The pure nonfactorizable corrections are negligible for almost the entire range, but do grow to $5-6 \%$ near the edge. Finally it is clear from the figure that the sub-leading terms are also small ( $\sim 2 \%$ ) for values of $M_{T}$ below $m_{t}$ and become more important for values beyond the edge.

The common characteristics shared by the contributions (4.3)-(4.5) for this observable can be understood by the fact that the distribution in the on-shell approach has a fixed edge at $m_{t}$ at LO, whilst at NLO it can only receive contributions beyond $m_{t}$ from events involving additional radiation that is clustered into $J_{b}$. In the off-shell computation (at both LO and NLO) this region receives additional contributions from events where $M_{\text {inv }}(t)>m_{t}$ and thus differs from the on-shell result distinctly. It should be noted that in this region soft-gluon resummation or parton shower effects are also expected to play an important role. 


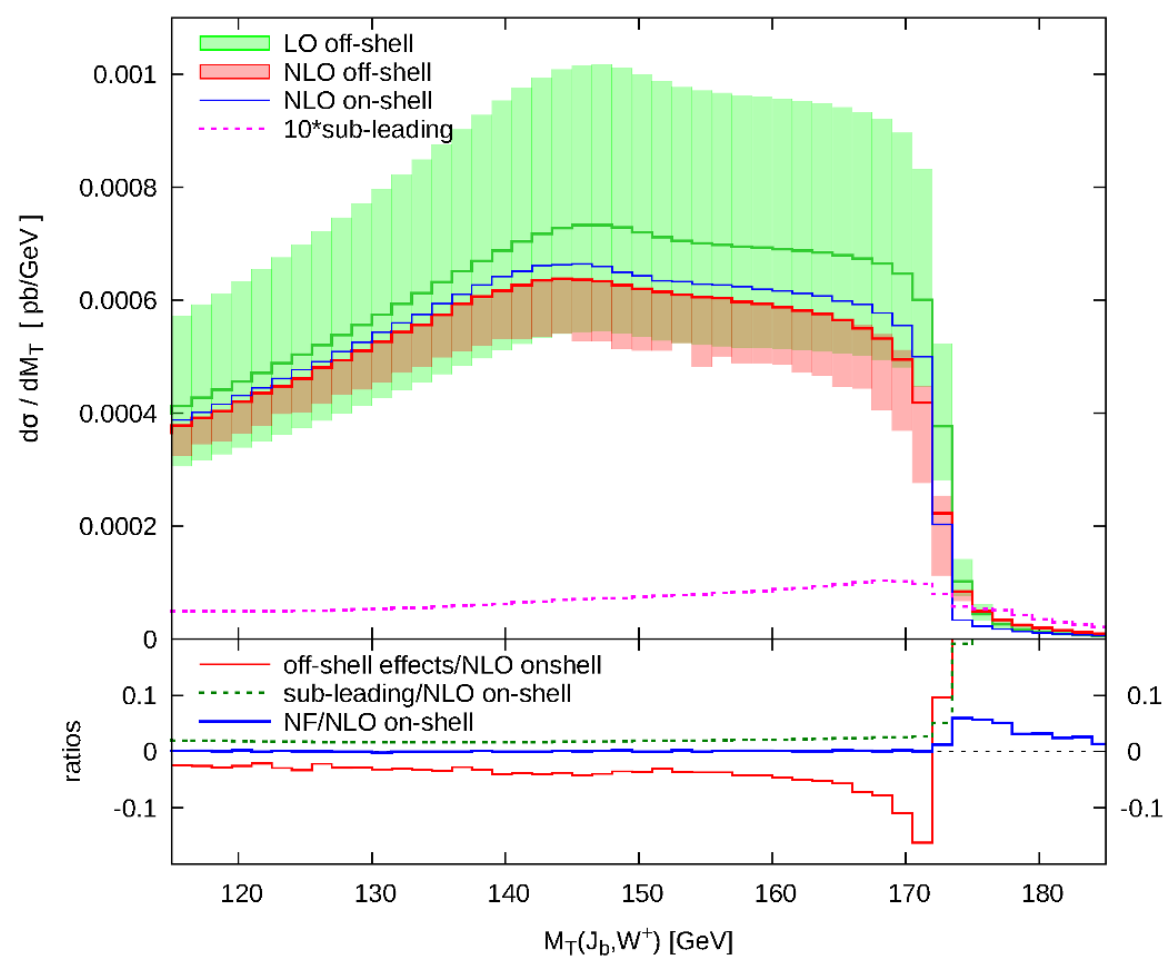

Figure 13. Transverse mass distribution for the reconstructed top quark. Upper plot: results for the off-shell LO result (green), off-shell NLO result (red) and on-shell NLO prediction (blue). Lower inset: relative contributions of total off-shell effects, non-factorizable corrections and singly-resonant diagrams. See the text for explanation.

\subsection{Individual NLO contributions}

We now examine the relative importance of the different contributions to the cross section for the distribution $H_{T}\left(J_{b}, J_{\bar{b}}\right)=p_{T}\left(J_{b}\right)+p_{T}\left(J_{\bar{b}}\right)$. The findings, illustrated in figure 14, are typical of observables that are inclusive in the invariant masses of the top quarks.

It is clear from the figure that the corrections to the top and antitop decay subprocesses are important for the correct normalization of the distribution, each separately correcting the LO results by about $-10 \%$. The negative sign of the latter means that there is a partial cancellation of NLO corrections between the production and decay subprocesses. It is evident from the almost flat shape of the decay corrections in the lower inset of figure 14 that these do not noticeably alter the shape of the distribution. Rather, any significant correction to the LO shape comes about through the factorizable corrections to the production subprocess. The non-factorizable corrections have not been included in the plot as they are tiny, as expected by the almost complete cancellation between real and virtual corrections for inclusive observables. In fact, the off-shell effects (also not displayed) are, as a whole, also small over the full range of $H_{T}$ considered, in agreement with the a priori expectation that these effects are of order $\sim \Gamma_{t} / m_{t}$. The sub-leading contributions constitute a $1-2 \%$ correction to the LO result (except at very low $H_{T}$ ), indicating that the EFT power-counting is working well. 


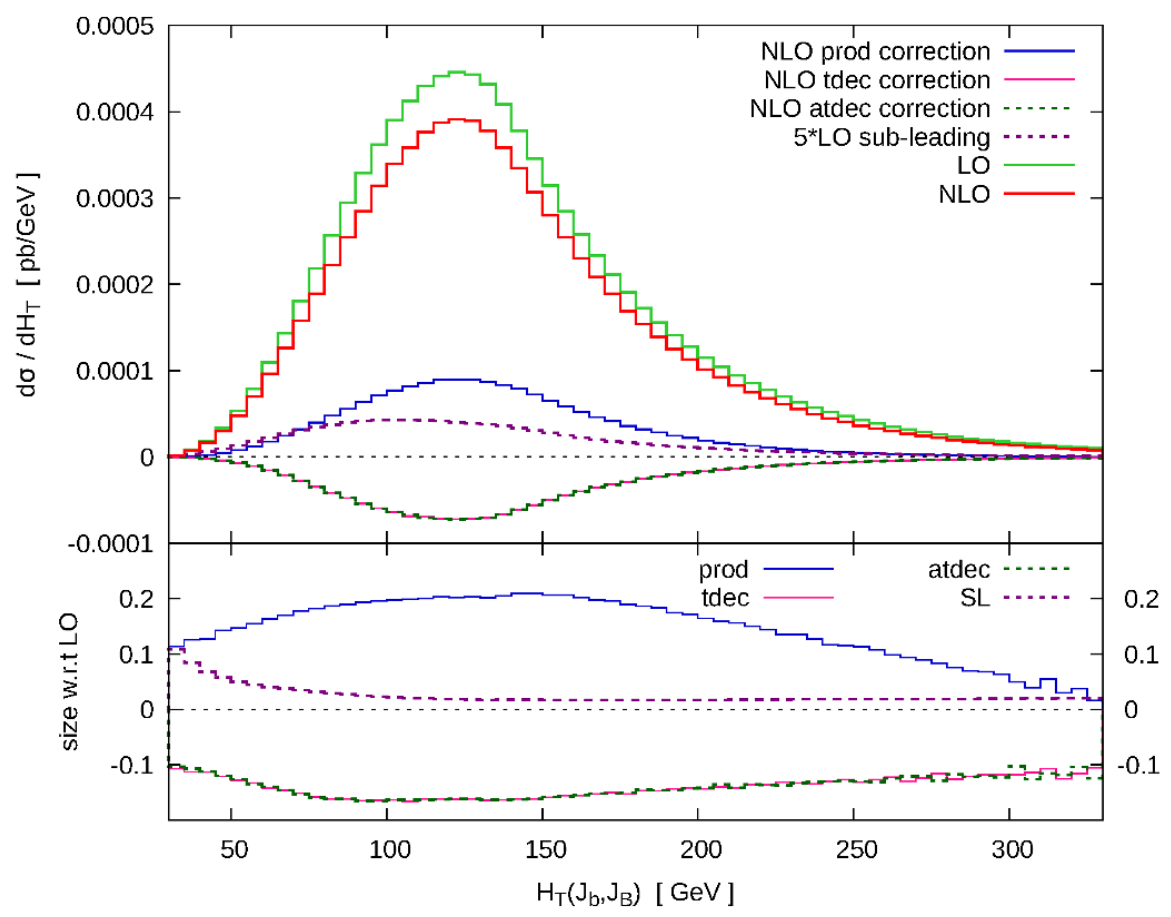

Figure 14. $H_{T}\left(J_{b}, J_{\bar{b}}\right)$ distribution. Upper plot: off-shell LO (green, solid) and NLO (red, solid) results. The factorizable corrections to the production (blue, solid), top decay (pink, solid) and antitop decay (dark green, dashed) subprocesses are also displayed along with the tree-level subleading contributions (purple, dashed). The ratios of the latter four contributions with respect to the LO results are shown in the lower inset (same colours and line-styles). See the text for explanation.

\subsection{Forward-backward asymmetry}

Figure 15 displays the differential distribution relevant for the study of the forwardbackward asymmetry of the top quark,

$$
\text { top asymmetry: } \quad \frac{d \sigma}{d y(t)}-\frac{d \sigma}{d y(\bar{t})} \text {. }
$$

The important feature to pick out is that off-shell effects amount to a small negative correction to the on-shell asymmetry of less than $4 \%$ in magnitude. This is fully expected for an observable that is inclusive over the invariant mass of the top. The sub-leading terms display a small asymmetry, giving a $\sim 7 \%$ correction to the on-shell asymmetry.

\subsection{Pole mass versus PS mass}

In this subsection we examine the effects of using the PS-mass scheme as an alternative to the pole scheme. As discussed previously, the PS mass has the advantage of being free of non-perturbative ambiguities whilst still being a suitable mass for the effective-theory setup as long as the choice $\mu_{\mathrm{PS}} \sim \alpha_{s} m_{t}$ is made.

We illustrate the above statements in figures 16 and 17 where the reconstructed topquark invariant mass and pseudo-rapidity are shown respectively. Distributions in the pole 


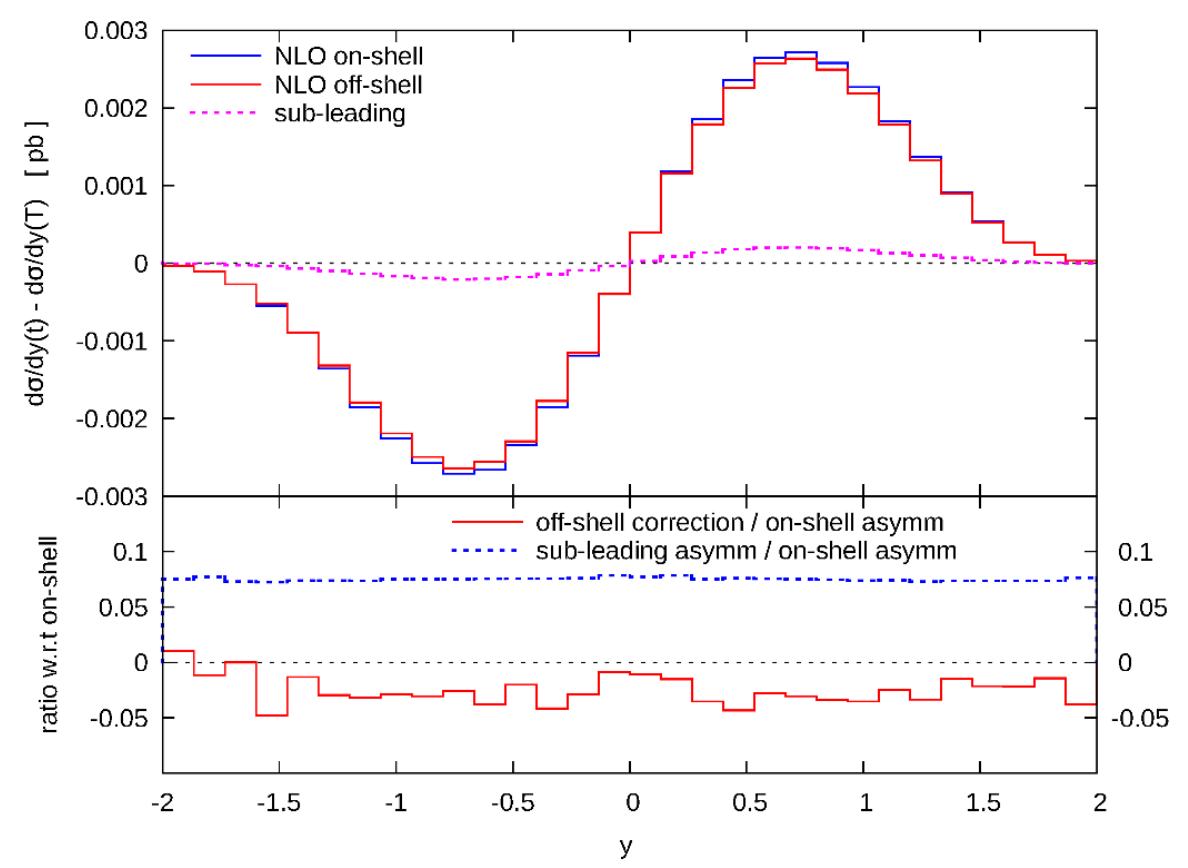

Figure 15. Differential forward-backward asymmetry for the reconstructed top quarks, see eq. (4.7). Upper plot: off-shell (red, solid), on-shell (blue, solid) and sub-leading (purple, dashed) asymmetries. Lower inset: ratio of the off-shell correction (red, solid) and sub-leading asymmetries (blue, dashed) with respect to the on-shell asymmetry. See text for further explanation.

scheme and PS-scheme with $\mu_{\mathrm{PS}} \in\{10,20,30,50\} \mathrm{GeV}$ at LO (1.h.s. panels) and NLO (r.h.s. panels) are plotted. In detail, we fix the numerical value of $m_{t, \mathrm{PS}}\left(\mu_{\mathrm{PS}}\right)$ to the pole mass $m_{t}=172.9 \mathrm{GeV}$ at next-to-leading order, i.e. including the term $\sim \delta_{1}^{\text {PS }}$ in eq. (3.21) and obtain $m_{t, \mathrm{PS}}(10)=172.44 \mathrm{GeV}, m_{t, \mathrm{PS}}(20)=171.97 \mathrm{GeV}, m_{t, \mathrm{PS}}(30)=171.53 \mathrm{GeV}$ and $m_{t, \mathrm{PS}}(50)=170.6 \mathrm{GeV}$ respectively. The width of the top is also adapted in accordance with $m_{t}$ at next-to-leading order in $\alpha_{s}$.

According to our discussion in sections 2.5 and 3.5, we would expect the suitability of $\mu_{\mathrm{PS}}=50 \mathrm{GeV}$ to be highly questionable, whereas $\mu_{\mathrm{PS}}=10 \mathrm{GeV}$ should be perfectly acceptable. To confirm this, firstly, we point out that at LO the distributions in all schemes agree very well with each other (within $\sim 5 \%$ for all bins), even for 'bad' scheme choices. This is clear since terms affecting the perturbative stability of a particular mass scheme only enter at orders beyond LO. In the RH panel of figure 16, where the NLO invariant mass distributions are plotted, it is evident that the choice $\mu_{\mathrm{PS}} \gtrsim 30 \mathrm{GeV}$ leads to serious deviations in shape from the pole-scheme curve and to large NLO corrections. This shift is due to the term given in eq. (3.25) and, to a lesser extent, due to non-factorizable corrections. The size of the NLO corrections implies that a NLO description of the lineshape for $\mu_{\mathrm{PS}} \gtrsim 30 \mathrm{GeV}$ is not trustworthy. In the same plot however, we observe that sensible scheme choices $\left(\mu_{\mathrm{PS}}=10,20 \mathrm{GeV}\right)$ give lineshapes that have a stable perturbative expansion. Thus it is perfectly legitimate to use such schemes rather than the pole-mass scheme for the extraction of the top mass from a measurement of the invariant mass of its decay products. Moreover, at NLO it is only observables that are more exclusive in the 

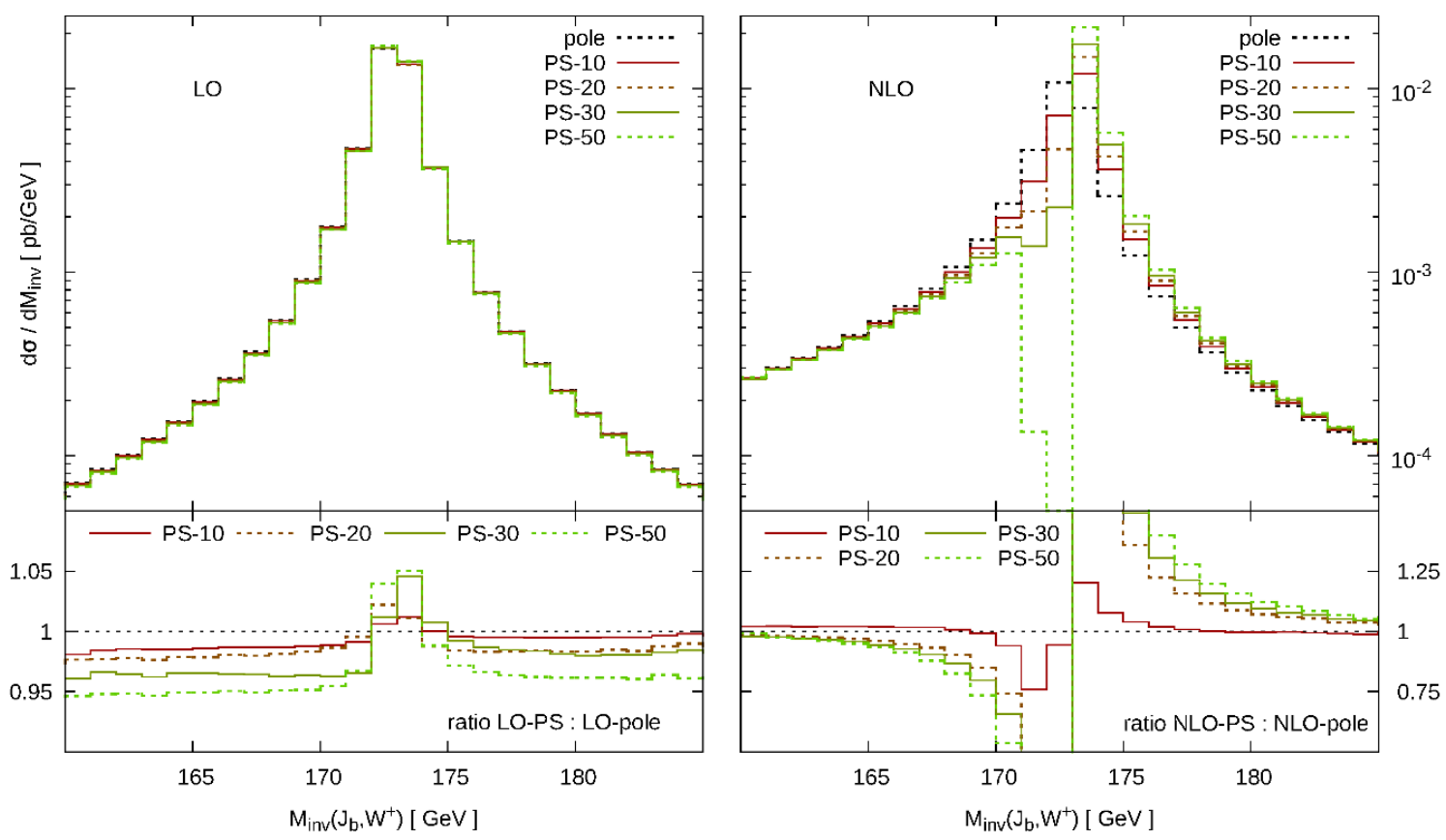

Figure 16. Invariant-mass distribution for reconstructed top quark at LO (left panel) and NLO (right panel) for pole and various PS-schemes. See the text for explanation.
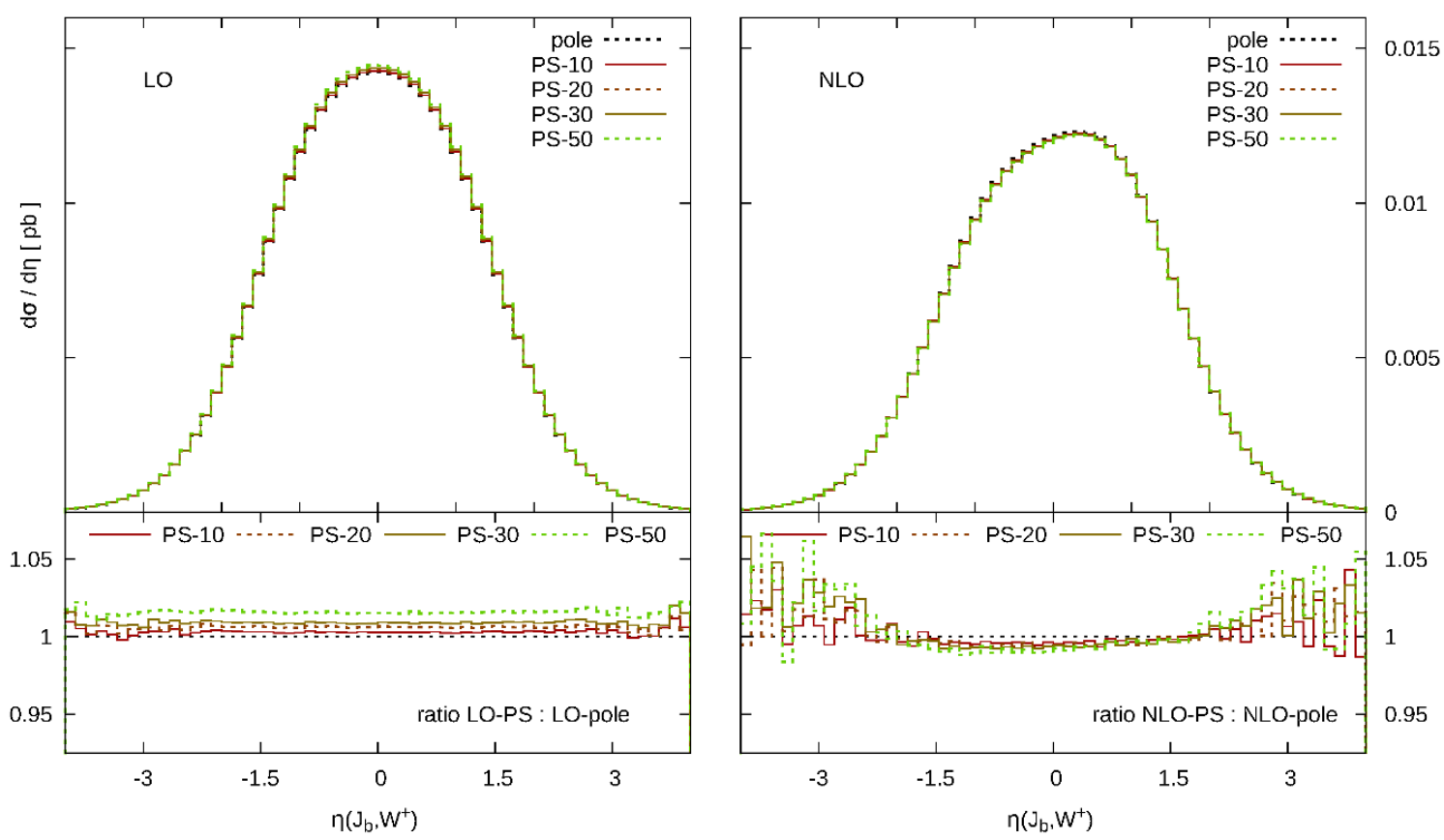

Figure 17. Pseudo-rapidity distribution for reconstructed top quark at LO (left panel) and NLO (right panel) for pole and various PS-schemes. See the text for explanation.

invariant mass that will display significant differences in shape for different schemes. The latter feature is illustrated well for the top pseudo-rapidity in the $\mathrm{RH}$ panel of figure 17, where all schemes display the same shape and the NLO corrections are under control. 


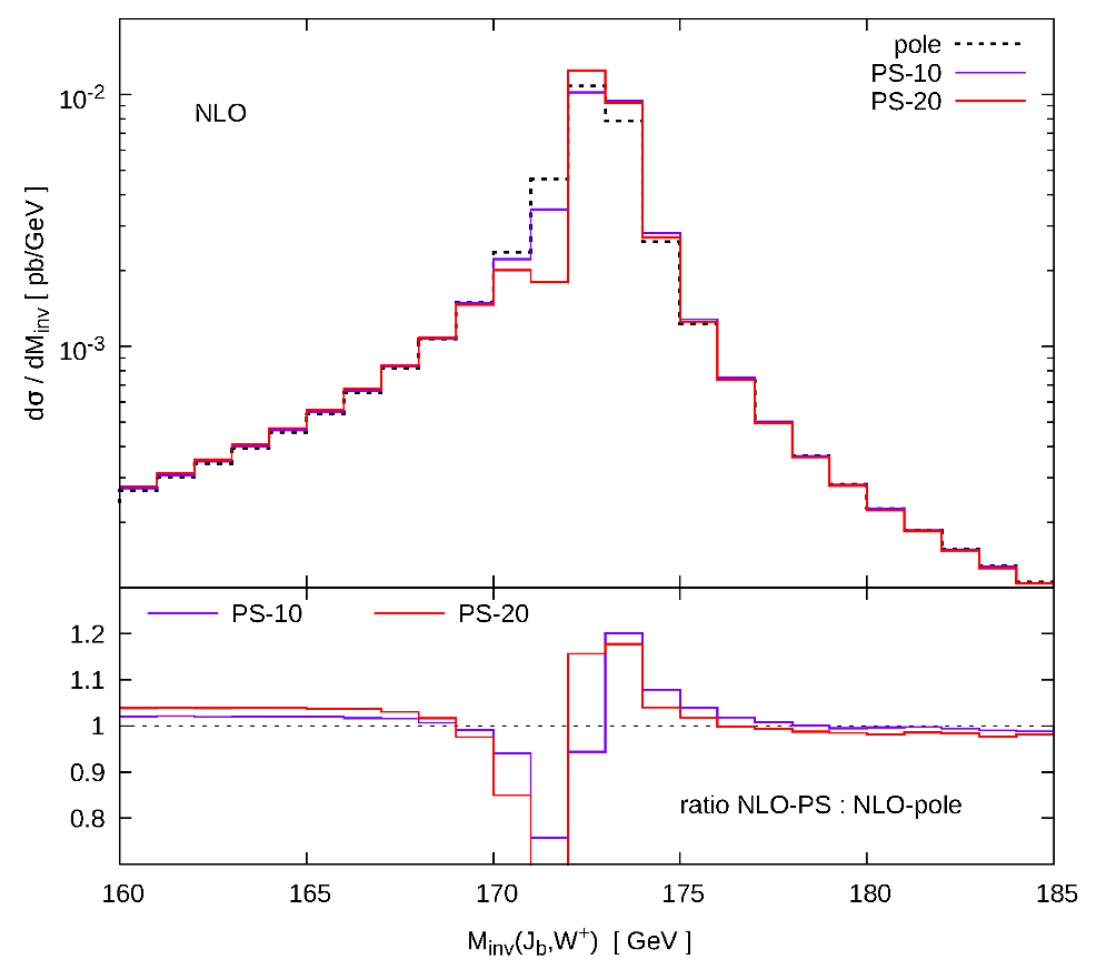

Figure 18. Invariant-mass distribution for reconstructed top quark at NLO for pole and various PS-schemes. See the text for explanation.

In order to investigate the impact of mass-scheme ambiguities on the determination of the top mass, we now consider in a toy analysis the invariant mass distribution for $\mu_{\mathrm{PS}} \in\{0,10,20\} \mathrm{GeV}$, where $\mu_{\mathrm{PS}}=0$ of course corresponds to the pole scheme. We want to compare the extraction of the top mass in these schemes at LO and NLO. We stress that we only consider effects from scheme changes and ignore all other effects, such as colour-reconnection effects and many more.

Starting at LO, we extract the mass in all schemes by adjusting its value to obtain optimal agreement with the measured distribution. Let us assume in the pole scheme the measured distribution is matched perfectly for $m_{t}=172.9 \mathrm{GeV}$. After the extraction of this value for the pole mass we can now convert the latter to the $\overline{\mathrm{MS}}$ scheme to obtain $\bar{m}=162.2$, where the conversion is done at three loop [74] with a crude Pade estimate ${ }^{7}$ for higher-order corrections. However, as we have argued above, we are also entitled to use the PS scheme with $\mu_{\mathrm{PS}}=10 \mathrm{GeV}$ or $\mu_{\mathrm{PS}}=20 \mathrm{GeV}$ for such an analysis. Given the results shown in the left panel of figure 16, the extracted values for the masses in these schemes are extremely close to the NLO converted masses given previously in this subsection and listed in the first column of table 2 . If we now convert these values to the $\overline{\mathrm{MS}}$ scheme with precisely the same procedure used for the pole scheme, we obtain the values for $\bar{m}$ listed in the second

\footnotetext{
${ }^{7}$ We emphasise that the precise method employed to estimate effects of higher order corrections does not play a major role here. For all numbers quoted in this subsection, including table 2, the estimate of the error in the conversion is $\lesssim 100 \mathrm{MeV}$.
} 


\begin{tabular}{|c|ccc|ccc|}
\hline & \multicolumn{3}{|c|}{ LO } & \multicolumn{3}{c|}{ NLO } \\
$\mu_{\mathrm{PS}}$ & $m_{\mathrm{e} x p}$ & $\bar{m}$ & $m_{t}$ & $m_{\mathrm{e} x p}$ & $\bar{m}$ & $m_{t}$ \\
\hline 0 & 172.9 & 162.2 & 172.9 & 172.9 & 162.2 & 172.9 \\
10 & 172.4 & 162.7 & 173.5 & 172.2 & 162.4 & 173.3 \\
20 & 172.0 & 163.0 & 173.8 & 171.5 & 162.5 & 173.4 \\
\hline
\end{tabular}

Table 2. Extraction of the top mass in various schemes at LO (left panel) and NLO (right panel). All numbers are in units of $\mathrm{GeV}$.

column of table 2 . These values differ by $600-800 \mathrm{MeV}$ from $\bar{m}=162.2$. Alternatively, if we convert the extracted values of $m_{t, \mathrm{PS}}(10)=172.44 \mathrm{GeV}$ and $m_{t, \mathrm{PS}}(20)=171.97 \mathrm{GeV}$ back to the pole scheme, we obtain the values $m_{t}=173.5 \mathrm{GeV}$ and $m_{t}=173.8 \mathrm{GeV}$ respectively, as listed in the third column of table 2. This conversion is done at $\mathcal{O}\left(\alpha_{s}^{4}\right)$, again supplemented by a crude Pade approximation to estimate higher-order effects. We stress that all values given in the left panel of table 2 are of course consistent at $\mathcal{O}\left(\alpha_{s}\right)$ and differ only by terms that are formally of higher order. The crucial point is that the numerical effect of these higher-order terms lead to an ambiguity of $500-900 \mathrm{MeV}$ in the LO extraction of the top mass from the invariant mass distribution.

We can now repeat this exercise at NLO where we begin by stressing that NLO in this context implies the inclusion of NLO corrections to the propagation of the top quark, in addition to the factorizable and non-factorizable corrections. Simply taking into account NLO corrections to the production subprocess alone does not improve the LO toy analysis presented above. We again assume that the experimental measurement of the invariant mass is perfectly matched in the pole scheme by setting $m_{t}=172.9 \mathrm{GeV}$. To extract the PS-mass from this measurement we would have to perform a best-fit analysis in the PSscheme and extract the best value for the top mass. We have found that this best value is very close (but not exactly identical) to the value obtained from two-loop conversion of the pole mass to the PS mass, listed in the first column in the right panel of table 2. In figure 18 we show the corresponding distributions to confirm that they are indeed very close.

As in the LO case we then convert the extracted values for the mass to the $\overline{\mathrm{MS}}$ scheme (second column) and back to the pole scheme (third column). Not surprisingly, the ambiguities in the extraction of the mass has decreased compared to the LO case and is now about $200-300 \mathrm{MeV}$ for the determination of $\bar{m}$ and $400-500 \mathrm{MeV}$ for the determination of the pole mass.

Let us conclude this subsection with a few comments regarding this toy analysis. First and foremost, this is of course only a basic investigation. Apart from taking into account all partonic channels, a fully rigorous analysis would have to include numerous effects beyond fixed order in perturbation theory. However, the main result that there is an existing additional ambiguity due to the scheme dependence, is unlikely to change drastically. In particular, it is not justified to blindly identify the extracted mass of the top quark as the pole mass. The size of this additional error will depend most of all on whether the analysis is done at LO or NLO. It also depends on what values of $\mu_{\mathrm{PS}}$ are considered acceptable - 
increasing the range in $\mu_{\mathrm{PS}}$ increases the spread in the extracted mass. We find that the ambiguity in the (indirect) determination of $\bar{m}$ is smaller than that present in the determination of the pole mass. This could be due to the better convergence of the associated perturbative series relating the different masses, which in turn is related to the renormalon ambiguities inherent to the pole mass.

\section{Conclusions}

We have presented an approach that allows the computation of cross sections involving heavy unstable particles, formalising the physical picture of production and subsequent decay. Our approach makes use of techniques common to effective-theory calculations, organizing the amplitude into contributions from matching coefficients and from dynamic soft degrees of freedom in the effective theory. This split allows one to disentangle and separately study effects from widely different scales. Compared to computations using the complex-mass scheme, our approach leads to calculations that are considerably simpler technically. However, our results do have the disadvantage of being valid only in the resonant region. To relax this constraint within the effective theory approach, we would have to match our results to a strict fixed-order calculation (without any self-energy resummation) outside the resonance region. Similarly, if we wanted to extend the results to the threshold region, we would have to match our effective theory to another NRQCD-like effective theory that describes a top pair near resonance.

The approach applied in this paper to top pair production from initial state quarks is a generalization and development of an approach applied earlier to single-top production. In particular, the real corrections are expanded at the integrand level so that no further expansions after phase space integration are required.

Generically, off-shell effects are found to be small and only have an impact near kinematic edges where distributions are not inclusive in the invariant mass and, therefore, the corresponding cancellations are not complete. As a toy application we have investigated the impact of mass-scheme ambiguities for the extraction of the top mass from the invariant mass distribution. In a simple analysis we found scheme ambiguities of $500-900 \mathrm{MeV}$ and $400-500 \mathrm{MeV}$ for the determination of the pole mass using LO and NLO calculations respectively. Future applications of the EFT method include the possibility of studying resummation of logarithms of ratios of widely separated scales for fully differential cross sections. The fact that effective theories disentangle effects from different scales is a promising starting point for such an investigation.

\section{Acknowledgments}

AP gratefully acknowledges the support and hospitality of the CERN and PSI Theory groups, where part of this work was completed. This research has been supported, in part, by the U.K. Science and Technology Facilities Council and the ERC grant 291377 "LHCtheory: theoretical predictions and analyses of LHC physics: advancing the precision 
frontier." The work of PF is supported by the "Stichting voor Fundamenteel Onderzook der Materie (FOM)".

Open Access. This article is distributed under the terms of the Creative Commons Attribution License which permits any use, distribution and reproduction in any medium, provided the original author(s) and source are credited.

\section{References}

[1] V.S. Fadin, V.A. Khoze and A.D. Martin, How suppressed are the radiative interference effects in heavy instable particle production?, Phys. Lett. B 320 (1994) 141 [hep-ph/9309234] [INSPIRE].

[2] D. Berdine, N. Kauer and D. Rainwater, Breakdown of the narrow width approximation for new physics, Phys. Rev. Lett. 99 (2007) 111601 [hep-ph/0703058] [INSPIRE].

[3] N. Kauer, Narrow-width approximation limitations, Phys. Lett. B 649 (2007) 413 [hep-ph/0703077] [INSPIRE].

[4] C. Uhlemann and N. Kauer, Narrow-width approximation accuracy, Nucl. Phys. B 814 (2009) 195 [arXiv: 0807.4112] [INSPIRE].

[5] J.M. Campbell, R.K. Ellis and C. Williams, Gluon-gluon contributions to $W^{+} W^{-}$production and Higgs interference effects, JHEP 10 (2011) 005 [arXiv:1107.5569] [INSPIRE].

[6] N. Kauer and G. Passarino, Inadequacy of zero-width approximation for a light Higgs boson signal, JHEP 08 (2012) 116 [arXiv:1206.4803] [INSPIRE].

[7] A. Denner, S. Dittmaier, M. Roth and L. Wieders, Electroweak corrections to charged-current $e^{+} e^{-} \rightarrow 4$ fermion processes: technical details and further results, Nucl. Phys. B 724 (2005) 247 [Erratum ibid. B 854 (2012) 504] [hep-ph/0505042] [INSPIRE].

[8] A. Denner and S. Dittmaier, The complex-mass scheme for perturbative calculations with unstable particles, Nucl. Phys. Proc. Suppl. 160 (2006) 22 [hep-ph/0605312] [INSPIRE].

[9] A. Bredenstein, A. Denner, S. Dittmaier and M. Weber, Radiative corrections to the semileptonic and hadronic Higgs-boson decays $H \rightarrow W W / Z Z \rightarrow 4$ fermions, JHEP 02 (2007) 080 [hep-ph/0611234] [INSPIRE].

[10] A. Denner, S. Dittmaier, S. Kallweit and S. Pozzorini, NLO QCD corrections to $W W b b$ production at hadron colliders, Phys. Rev. Lett. 106 (2011) 052001 [arXiv:1012.3975] [INSPIRE].

[11] G. Bevilacqua, M. Czakon, A. van Hameren, C.G. Papadopoulos and M. Worek, Complete off-shell effects in top quark pair hadroproduction with leptonic decay at next-to-leading order, JHEP 02 (2011) 083 [arXiv:1012.4230] [INSPIRE].

[12] A. Denner, S. Dittmaier, S. Kallweit and S. Pozzorini, NLO QCD corrections to off-shell top-antitop production with leptonic decays at hadron colliders, JHEP 10 (2012) 110 [arXiv:1207.5018] [INSPIRE].

[13] P. Falgari, P. Mellor and A. Signer, Production-decay interferences at NLO in QCD for t-channel single-top production, Phys. Rev. D 82 (2010) 054028 [arXiv:1007.0893] [INSPIRE]. 
[14] P. Falgari, F. Giannuzzi, P. Mellor and A. Signer, Off-shell effects for t-channel and s-channel single-top production at NLO in QCD, Phys. Rev. D 83 (2011) 094013 [arXiv: 1102.5267] [INSPIRE].

[15] M. Beneke, A. Chapovsky, A. Signer and G. Zanderighi, Effective theory calculation of resonant high-energy scattering, Nucl. Phys. B 686 (2004) 205 [hep-ph/0401002] [InSPIRE].

[16] M. Beneke, P. Falgari, C. Schwinn, A. Signer and G. Zanderighi, Four-fermion production near the W-pair production threshold, Nucl. Phys. B 792 (2008) 89 [arXiv:0707.0773] [INSPIRE].

[17] S. Actis, M. Beneke, P. Falgari and C. Schwinn, Dominant NNLO corrections to four-fermion production near the W-pair production threshold, Nucl. Phys. B 807 (2009) 1 [arXiv: 0807.0102] [INSPIRE].

[18] R.G. Stuart, Gauge invariance, analyticity and physical observables at the $Z^{0}$ resonance, Phys. Lett. B 262 (1991) 113 [INSPIRE].

[19] A. Aeppli, G.J. van Oldenborgh and D. Wyler, Unstable particles in one loop calculations, Nucl. Phys. B 428 (1994) 126 [hep-ph/9312212] [INSPIRE].

[20] SM AND NLO MULTILEG and SM MC Working GRoups collaborations,

J. Alcaraz Maestre et al., The SM and NLO Multileg and SM MC Working Groups: summary report, arXiv: 1203.6803 [INSPIRE].

[21] M. Beneke and V.A. Smirnov, Asymptotic expansion of Feynman integrals near threshold, Nucl. Phys. B 522 (1998) 321 [hep-ph/9711391] [INSPIRE].

[22] A. Chapovsky, V.A. Khoze, A. Signer and W.J. Stirling, Nonfactorizable corrections and effective field theories, Nucl. Phys. B 621 (2002) 257 [hep-ph/0108190] [InSPIRE].

[23] J.M. Campbell, R.K. Ellis and F. Tramontano, Single top production and decay at next-to-leading order, Phys. Rev. D 70 (2004) 094012 [hep-ph/0408158] [InSPIRE].

[24] I.I. Bigi, M.A. Shifman, N. Uraltsev and A. Vainshtein, The pole mass of the heavy quark. Perturbation theory and beyond, Phys. Rev. D 50 (1994) 2234 [hep-ph/9402360] [INSPIRE].

[25] M. Beneke and V.M. Braun, Heavy quark effective theory beyond perturbation theory: renormalons, the pole mass and the residual mass term, Nucl. Phys. B 426 (1994) 301 [hep-ph/9402364] [INSPIRE].

[26] M.C. Smith and S.S. Willenbrock, Top quark pole mass, Phys. Rev. Lett. 79 (1997) 3825 [hep-ph/9612329] [INSPIRE].

[27] M. Beneke, A quark mass definition adequate for threshold problems, Phys. Lett. B 434 (1998) 115 [hep-ph/9804241] [INSPIRE].

[28] A. Hoang and T. Teubner, Top quark pair production close to threshold: top mass, width and momentum distribution, Phys. Rev. D 60 (1999) 114027 [hep-ph/9904468] [INSPIRE].

[29] A. Pineda, Determination of the bottom quark mass from the $\Upsilon(1 S)$ system, JHEP 06 (2001) 022 [hep-ph/0105008] [INSPIRE].

[30] S. Fleming, A.H. Hoang, S. Mantry and I.W. Stewart, Jets from massive unstable particles: top-mass determination, Phys. Rev. D 77 (2008) 074010 [hep-ph/0703207] [INSPIRE].

[31] S. Fleming, A.H. Hoang, S. Mantry and I.W. Stewart, Top jets in the peak region: factorization analysis with NLL resummation, Phys. Rev. D 77 (2008) 114003 [arXiv:0711.2079] [INSPIRE]. 
[32] P. Nason, S. Dawson and R.K. Ellis, The total cross-section for the production of heavy quarks in hadronic collisions, Nucl. Phys. B 303 (1988) 607 [INSPIRE].

[33] P. Nason, S. Dawson and R.K. Ellis, The one particle inclusive differential cross-section for heavy quark production in hadronic collisions, Nucl. Phys. B 327 (1989) 49 [Erratum ibid. B 335 (1990) 260] [INSPIRE].

[34] W. Beenakker, W. van Neerven, R. Meng, G. Schuler and J. Smith, QCD corrections to heavy quark production in hadron hadron collisions, Nucl. Phys. B 351 (1991) 507 [INSPIRE].

[35] M.L. Mangano, P. Nason and G. Ridolfi, Heavy quark correlations in hadron collisions at next-to-leading order, Nucl. Phys. B 373 (1992) 295 [INSPIRE].

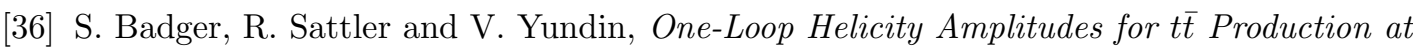
Hadron Colliders, Phys. Rev. D 83 (2011) 074020 [arXiv:1101.5947] [InSPIRE].

[37] W. Beenakker et al., Electroweak one loop contributions to top pair production in hadron colliders, Nucl. Phys. B 411 (1994) 343 [INSPIRE].

[38] S. Moretti, M. Nolten and D. Ross, Weak corrections to gluon-induced top-antitop hadro-production, Phys. Lett. B 639 (2006) 513 [Erratum ibid. B 660 (2008) 607] [hep-ph/0603083] [INSPIRE].

[39] J.H. Kuhn, A. Scharf and P. Uwer, Electroweak effects in top-quark pair production at hadron colliders, Eur. Phys. J. C 51 (2007) 37 [hep-ph/0610335] [InSPIRE].

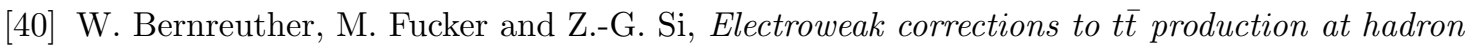
colliders, Nuovo Cim. B 123 (2008) 1036 [arXiv:0808.1142] [INSPIRE].

[41] W. Hollik and M. Kollar, NLO QED contributions to top-pair production at hadron collider, Phys. Rev. D 77 (2008) 014008 [arXiv:0708.1697] [InSPIRE].

[42] M. Czakon, A. Mitov and S. Moch, Heavy-quark production in massless quark scattering at two loops in QCD, Phys. Lett. B 651 (2007) 147 [arXiv:0705.1975] [InSPIRE].

[43] M. Czakon, A. Mitov and S. Moch, Heavy-quark production in gluon fusion at two loops in QCD, Nucl. Phys. B 798 (2008) 210 [arXiv:0707.4139] [InSPIRE].

[44] S. Dittmaier, P. Uwer and S. Weinzierl, $N L O$ QCD corrections to $t \bar{t}+$ jet production at hadron colliders, Phys. Rev. Lett. 98 (2007) 262002 [hep-ph/0703120] [INSPIRE].

[45] S. Dittmaier, P. Uwer and S. Weinzierl, Hadronic top-quark pair production in association with a hard jet at next-to-leading order QCD: phenomenological studies for the Tevatron and the LHC, Eur. Phys. J. C 59 (2009) 625 [arXiv:0810.0452] [inSPIRE].

[46] M. Czakon, Tops from light quarks: full mass dependence at two-loops in QCD, Phys. Lett. B 664 (2008) 307 [arXiv:0803.1400] [INSPIRE].

[47] C. Anastasiou and S.M. Aybat, The one-loop gluon amplitude for heavy-quark production at NNLO, Phys. Rev. D 78 (2008) 114006 [arXiv:0809.1355] [INSPIRE].

[48] R. Bonciani, A. Ferroglia, T. Gehrmann, D. Maître and C. Studerus, Two-loop fermionic corrections to heavy-quark pair production: the quark-antiquark channel, JHEP 07 (2008) 129 [arXiv: 0806.2301] [INSPIRE].

[49] R. Bonciani, A. Ferroglia, T. Gehrmann and C. Studerus, Two-loop planar corrections to heavy-quark pair production in the quark-antiquark channel, JHEP 08 (2009) 067 [arXiv:0906.3671] [INSPIRE]. 
[50] K. Melnikov and M. Schulze, NLO QCD corrections to top quark pair production in association with one hard jet at hadron colliders, Nucl. Phys. B 840 (2010) 129 [arXiv: 1004.3284] [INSPIRE].

[51] M. Czakon, A novel subtraction scheme for double-real radiation at NNLO, Phys. Lett. B 693 (2010) 259 [arXiv: 1005.0274] [INSPIRE].

[52] R. Bonciani, A. Ferroglia, T. Gehrmann, A. Manteuffel and C. Studerus, Two-loop leading color corrections to heavy-quark pair production in the gluon fusion channel, JHEP 01 (2011) 102 [arXiv:1011.6661] [INSPIRE].

[53] I. Bierenbaum, M. Czakon and A. Mitov, The singular behavior of one-loop massive QCD amplitudes with one external soft gluon, Nucl. Phys. B 856 (2012) 228 [arXiv:1107.4384] [INSPIRE].

[54] P. Baernreuther, M. Czakon and A. Mitov, Percent level precision physics at the Tevatron: first genuine NNLO QCD corrections to $q \bar{q} \rightarrow t \bar{t}+X$, Phys. Rev. Lett. 109 (2012) 132001 [arXiv: 1204.5201] [INSPIRE].

[55] M. Czakon and A. Mitov, NNLO corrections to top-pair production at hadron colliders: the all-fermionic scattering channels, JHEP 12 (2012) 054 [arXiv:1207.0236] [INSPIRE].

[56] M. Czakon and A. Mitov, NNLO corrections to top pair production at hadron colliders: the quark-gluon reaction, JHEP 01 (2013) 080 [arXiv:1210.6832] [INSPIRE].

[57] N. Kidonakis, Next-to-next-to-leading soft-gluon corrections for the top quark cross section and transverse momentum distribution, Phys. Rev. D 82 (2010) 114030 [arXiv:1009.4935] [INSPIRE].

[58] V. Ahrens, A. Ferroglia, M. Neubert, B.D. Pecjak and L.-L. Yang, RG-improved single-particle inclusive cross sections and forward-backward asymmetry in t $\bar{t}$ production at hadron colliders, JHEP 09 (2011) 070 [arXiv:1103.0550] [INSPIRE].

[59] M. Cacciari, M. Czakon, M. Mangano, A. Mitov and P. Nason, Top-pair production at hadron colliders with next-to-next-to-leading logarithmic soft-gluon resummation, Phys. Lett. B 710 (2012) 612 [arXiv:1111.5869] [INSPIRE].

[60] M. Beneke, P. Falgari, S. Klein and C. Schwinn, Hadronic top-quark pair production with NNLL threshold resummation, Nucl. Phys. B 855 (2012) 695 [arXiv:1109.1536] [INSPIRE].

[61] M. Beneke, P. Falgari, S. Klein, J. Piclum and C. Schwinn, NNLL threshold resummation for the total top-pair production cross section, arXiv:1205.0988 [INSPIRE].

[62] W. Bernreuther, A. Brandenburg, Z. Si and P. Uwer, Top quark pair production and decay at hadron colliders, Nucl. Phys. B 690 (2004) 81 [hep-ph/0403035] [INSPIRE].

[63] K. Melnikov and M. Schulze, NLO QCD corrections to top quark pair production and decay at hadron colliders, JHEP 08 (2009) 049 [arXiv: 0907.3090] [INSPIRE].

[64] W. Bernreuther and Z.-G. Si, Distributions and correlations for top quark pair production and decay at the Tevatron and LHC., Nucl. Phys. B 837 (2010) 90 [arXiv:1003.3926] [InSPIRE].

[65] J.M. Campbell and R.K. Ellis, Top-quark processes at NLO in production and decay, arXiv: 1204.1513 [INSPIRE].

[66] J. Gao, C.S. Li and H.X. Zhu, Top quark decay at next-to-next-to leading order in QCD, Phys. Rev. Lett. 110 (2013) 042001 [arXiv: 1210.2808] [INSPIRE]. 
[67] M. Brucherseifer, F. Caola and K. Melnikov, $\mathcal{O}\left(\alpha_{s}^{2}\right)$ corrections to fully-differential top quark decays, JHEP 04 (2013) 059 [arXiv: 1301.7133] [INSPIRE].

[68] S. Frixione, Z. Kunszt and A. Signer, Three jet cross-sections to next-to-leading order, Nucl. Phys. B 467 (1996) 399 [hep-ph/9512328] [INSPIRE].

[69] R. Frederix, S. Frixione, F. Maltoni and T. Stelzer, Automation of next-to-leading order computations in QCD: the FKS subtraction, JHEP 10 (2009) 003 [arXiv:0908.4272] [INSPIRE].

[70] S. Catani and M. Seymour, A general algorithm for calculating jet cross-sections in NLO QCD, Nucl. Phys. B 485 (1997) 291 [Erratum ibid. B 510 (1998) 503] [hep-ph/9605323] [INSPIRE].

[71] S. Catani, S. Dittmaier, M.H. Seymour and Z. Trócsányi, The dipole formalism for next-to-leading order QCD calculations with massive partons, Nucl. Phys. B 627 (2002) 189 [hep-ph/0201036] [INSPIRE].

[72] A. Denner, S. Dittmaier, M. Roth and D. Wackeroth, Electroweak radiative corrections to $e^{+} e^{-} \rightarrow W W \rightarrow 4$ fermions in double pole approximation: the RACOONWW approach, Nucl. Phys. B 587 (2000) 67 [hep-ph/0006307] [InSPIRE].

[73] A. Martin, W. Stirling, R. Thorne and G. Watt, Parton distributions for the LHC, Eur. Phys. J. C 63 (2009) 189 [arXiv:0901.0002] [InSPIRE].

[74] K. Melnikov and T.v. Ritbergen, The three loop relation between the MS-bar and the pole quark masses, Phys. Lett. B 482 (2000) 99 [hep-ph/9912391] [INSPIRE]. 